\title{
Facile Preparation of New Optically Pure 7,7'-disubstituted BINOLs and Their Application in Asymmetric Catalysis
}

\author{
Quan-zhong Liu, Nan-Sheng Xie, Zhi-Bin Luo ${ }^{\dagger}$, Xin Cui, \\ Lin-Feng Cun ${ }^{\dagger}$, Liu-Zhu Gong*, Ai-Qiao Mi ${ }^{\dagger}$, Yao-Zhong Jiang* \\ Key Laboratory for Asymmetric Synthesis and Chirotechnology of Sichuan Province, Chengdu Institute of Organic \\ Chemistry, Chinese Academy of Sciences, Chengdu, 610041, China, and Chemistry Department, Sichuan Normal \\ College, Nanchong, 637002, China \\ gonglz@cioc.ac.cn
}

(Supplementary Materials)

Table of Contents

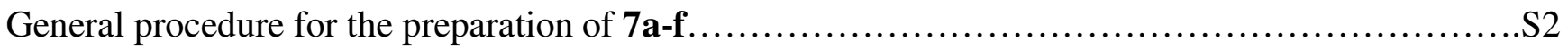

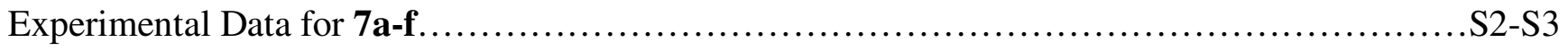

General procedure for the asymmetric addition of phenylacetylene to aromatic aldehyde..........S3-S4

General procedure for the asymmetric addition of phenylacetylene to aliphatic aldehyde............S4

Experimental Data for asymmetric addition of phenylacetylene to aldehydes...................S4-S6

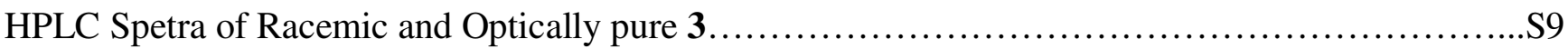

Copy of ${ }^{1} \mathrm{HNMR}$ and ${ }^{13} \mathrm{CNMR}$ spectra of

3 (S7, S8), 5 (S10, S11), 7a (S12, S13), 7b (S14, S15), 7c (S16, S17), 7d (S18, S19), 7e (S20, S21), 7f

(S22, S23), 8a (S24, S25), 8b (S26, S27), 8c (S28, S29), 9a (S30, S31), 9b (S32, S33), 11(S34, S35) 


\section{General procedure for the synthesis of 7,7'-dialkoxy-2,2'-dihydroxy-1,1'- dinaphthyl (7a-7f):}

5 (121.8 mg, $0.3 \mathrm{mmol}), \mathrm{CsOH} \bullet \mathrm{H}_{2} \mathrm{O}(101 \mathrm{mg}, 0.6 \mathrm{mmol})$ and alkyl bromide $(0.6 \mathrm{mmol})$ were dissolved in DMF $(50 \mathrm{~mL})$. After the solution was stirred at $80{ }^{\circ} \mathrm{C}$ for 2 hours, water $(100 \mathrm{ml})$ was added. The aqueous solution was extracted with ethyl acetate $(100 \mathrm{~mL})$, the combined organic layer was washed with brine and dried over anhydrous $\mathrm{Na}_{2} \mathrm{SO}_{4}$. After removal of solvent, the residue was dissolved in a mixture of chloroform $(25 \mathrm{~mL}), 6 \mathrm{~N} \mathrm{HCl}(25 \mathrm{~mL})$ and ethanol $(10 \mathrm{~mL})$, and then refluxed for 2 hours. The chloroform layer was separated, and the aqueous layer was extracted with chloroform $(25 \mathrm{~mL})$. The combined organic layer was concentrated and purified by column chromatography on silica gel to give 7a-7f.

7a, $R_{\mathrm{f}}=0.43$ (ethyl acetate/petroleum $=1: 3$ ), yield: $91 \%$, white solid, $\mathrm{mp} 136-137^{\circ} \mathrm{C},[\alpha]_{\mathrm{D}}{ }^{20}=-146.7^{\circ},(\mathrm{c}$ $\left.=0.36, \mathrm{CHCl}_{3}\right)\left(\mathrm{lit}^{5 \mathrm{j}}:[\alpha]_{\mathrm{D}}{ }^{20}=-126.4^{\mathrm{o}},\left(\mathrm{c}=1.0, \mathrm{CH}_{3} \mathrm{OH}\right) .{ }^{1} \mathrm{HNMR}\left(300 \mathrm{MHz}, \mathrm{CDCl}_{3}\right) \delta(\mathrm{ppm}) 3.58(\mathrm{~s}\right.$, 6H) $5.08(\mathrm{~s}, 2 \mathrm{H}), 6.49(\mathrm{~d}, J=2.4 \mathrm{~Hz}, 2 \mathrm{H}), 7.03(\mathrm{dd}, J=9.0 \mathrm{~Hz}, 2.4 \mathrm{~Hz}, 2 \mathrm{H}), 7.22(\mathrm{dd}, J=8.8 \mathrm{~Hz}, 2 \mathrm{H})$, $7.78(\mathrm{~d}, J=9.0 \mathrm{~Hz}, 2 \mathrm{H}), 7.87(\mathrm{~d}, J=8.8 \mathrm{~Hz}, 2 \mathrm{H}),{ }^{13} \mathrm{CNMR}\left(75 \mathrm{MHz}, \mathrm{CDCl}_{3}\right) \quad \delta(\mathrm{ppm}) 55.1,103.1,110.0$, 115.1, 116.0, 124.8, 130.0, 131.1, 134.7, 153.3, 159.1; MS (EI) m/z 346 (M+); HRESI-MS(positive ion) $\mathrm{C}_{22} \mathrm{H}_{19} \mathrm{O}_{4}\left([\mathrm{M}+\mathrm{H}]^{+}\right)$requires 347.1283, found 347.1278. IR (KBr) v 3444, 3062, 2937, 2835, 1621, 1513, 1463, 1221, $1032 \mathrm{~cm}^{-1}$. Anal. Calca for $\mathrm{C}_{22} \mathrm{H}_{218} \mathrm{O}_{4}$ : C, 76.29; H, 5.24. Found: C, 76.56; H, 5.26.

7b, $R_{\mathrm{f}}=0.58$ (ethyl acetate/petroleum $=1: 3$ ), yield: $91 \%$, white solid, $\mathrm{mp} 69.0-70.3^{\circ} \mathrm{C} ;[\alpha]_{\mathrm{D}}{ }^{20}=-196.3^{\circ}$, $\left(\mathrm{c}=0.108, \mathrm{CHCl}_{3}\right) ;{ }^{1} \mathrm{HNMR}\left(300 \mathrm{MHz}, \mathrm{CDCl}_{3}\right), \delta(\mathrm{ppm}) 1.27(\mathrm{t}, J=7.0 \mathrm{~Hz}, 6 \mathrm{H}), 3.67-3.86(\mathrm{~m}, 4 \mathrm{H})$, 5.06 (brs, 2H), 6.48 (d, J=1.9 Hz, 2H), 7.03 (dd, J=8.9, 2.3 Hz, 2H), 7.20 (d, J=8.8 Hz, 2H), 7.77 (d, J= $8.9 \mathrm{~Hz}, 2 \mathrm{H}), 7.87(\mathrm{~d}, J=8.8 \mathrm{~Hz}, 2 \mathrm{H}) ;{ }^{13} \mathrm{CNMR}\left(75 \mathrm{MHz}, \mathrm{CDCl}_{3}\right) \delta(\mathrm{ppm}) 14.5,63.3,103.9,109.9,115.0$, 116.2, 124.7, 129.9, 131.1, 134.7, 153.2, 158.4; MS (EI) m/z 374 (M+); HRESI-MS(positive ion) $\mathrm{C}_{24} \mathrm{H}_{23} \mathrm{O}_{4}\left([\mathrm{M}+\mathrm{H}]^{+}\right)$requires 375.1596, found 375.1591. IR (KBr) v 3427, 3061, 2977, 2931, 1620, 1513, 1216, $1042 \mathrm{~cm}^{-1}$. Anal. Calca for $\mathrm{C}_{24} \mathrm{H}_{22} \mathrm{O}_{4}$ : C, 76.99; H, 5.92. Found: C, 76.80; H, 5.79.

7c, $\mathrm{R}_{\mathrm{f}}=0.29$ (ethyl acetate/petroleum $=1: 5$ ), yield: $93 \%$, colourless sticky oil, $[\alpha]_{\mathrm{D}}{ }^{20}=-192.7^{\mathrm{o}},(\mathrm{c}=$

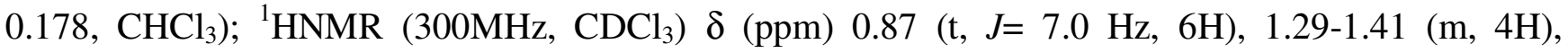
1.57-1.66 (m, 4H), 3.65-3.77 (m, 4H), 5.04 (brs, 2H), 6.49 (d, J=2.4 Hz, 2H), 7.03 (dd, J= 8.9, 2.4 Hz, 2H), $7.18(\mathrm{~d}, J=8.8 \mathrm{~Hz}, 2 \mathrm{H}), 7.76(\mathrm{~d}, J=8.9 \mathrm{~Hz}, 2 \mathrm{H}), 7.83(\mathrm{~d}, J=8.8 \mathrm{~Hz}, 2 \mathrm{H}) ;{ }^{13} \mathrm{CNMR}(75 \mathrm{MHz}$, $\left.\mathrm{CDCl}_{3}\right) \delta(\mathrm{ppm}) 13.6,19.0,30.9,67.3,103.9,110.0,114.9,116.2,124.6,129.8,130.9,134.7,153.2$, 158.5; MS (EI) m/z $430\left(\mathrm{M}^{+}\right)$; HRESI-MS(positive ion) $\mathrm{C}_{28} \mathrm{H}_{31} \mathrm{O}_{4}\left([\mathrm{M}+\mathrm{H}]^{+}\right)$requires 431.2222, found 431.2217. IR (KBr) v 3477, 3061, 2958, 2872, 1620, 1513, 1454, 1216, $1027 \mathrm{~cm}^{-1}$. Anal. Calcd for $\mathrm{C}_{28} \mathrm{H}_{30} \mathrm{O}_{4}$ : C, 76.11; H, 7.02. Found: C, 76.30; H, 7.18. 
7d, $R_{\mathrm{f}}=0.30$ (ethyl acetate/petroleum $\left.=1: 5\right)$, Yield: $92 \%$, colourless sticky oil, $[\alpha]_{\mathrm{D}}{ }^{20}=-224.5^{\circ},(\mathrm{c}=$ 0.144, $\left.\mathrm{CHCl}_{3}\right) ;{ }^{1} \mathrm{HNMR}\left(300 \mathrm{MHz}, \mathrm{CDCl}_{3}\right) \delta(\mathrm{ppm}) 0.86(\mathrm{t}, J=6.6 \mathrm{~Hz}, 6 \mathrm{H}), 1.21-1.34(\mathrm{~m}, 12 \mathrm{H})$, 1.59-1.64 (m, 4H), 3.66-3.76 (m, 4H), 5.08 (brs, 2H), 6.48 (d, J=2.4 Hz, 2H), 7.03 (dd, J= 8.8 Hz, 2.4 $\mathrm{Hz}, 2 \mathrm{H}), 7.19(\mathrm{~d}, J=8.8 \mathrm{~Hz}, 2 \mathrm{H}), 7.77(\mathrm{~d}, J=8.8 \mathrm{~Hz}, 2 \mathrm{H}), 7.85(\mathrm{~d}, J=8.8 \mathrm{~Hz}, 2 \mathrm{H}),{ }^{13} \mathrm{CNMR}(75 \mathrm{MHz}$, $\left.\mathrm{CDCl}_{3}\right) \delta(\mathrm{ppm}) 14.0,22.5,25.6,28.9,31.5,67.8,103.9,110.0,114.9,116.3,124.6,129.8,131.0,134.7$, 153.2, 158.5; MS (EI) m/z $486\left(\mathrm{M}^{+}\right)$; HRESI-MS(positive ion) $\mathrm{C}_{32} \mathrm{H}_{39} \mathrm{O}_{4}\left([\mathrm{M}+\mathrm{H}]^{+}\right.$) requires 487.2848, found 487.4823. IR (KBr) v 3474, 3060, 2954, 2860, 1620, 1513, 1454, 1216, $1024 \mathrm{~cm}^{-1}$. Anal. Calcd. for $\mathrm{C}_{32} \mathrm{H}_{38} \mathrm{O}_{4}$ : C, 78.98; H, 7.87. Found: C, 79.06; H, 7.80.

7e, $R_{\mathrm{f}}=0.30$ (ethyl acetate/petroleum $\left.=1: 10\right)$, yield: $85 \%$, colourless sticky oil, $[\alpha]_{\mathrm{D}}{ }^{20}=-165.1^{\mathrm{o}},(\mathrm{c}=$ 0.146, $\left.\mathrm{CHCl}_{3}\right) ;{ }^{1} \mathrm{HNMR}\left(300 \mathrm{MHz}, \mathrm{CDCl}_{3}\right) \delta(\mathrm{ppm}) 0.91(\mathrm{t}, J=6.9 \mathrm{~Hz}, 6 \mathrm{H}), 1.27-1.32(\mathrm{~m}, 20 \mathrm{H})$, 1.59-1.69 (m, 4H), 3.65-3.79 (m, 4H), 4.88 (brs, 2H), 6.50 (d, J=2.4 Hz, 2H), 7.04 (dd, J= 8.9 Hz, 2.4 $\mathrm{Hz}, 2 \mathrm{H}), 7.17(\mathrm{~d}, J=8.8 \mathrm{~Hz}, 2 \mathrm{H}), 7.76(\mathrm{~d}, J=8.9 \mathrm{~Hz}, 2 \mathrm{H}), 7.82(\mathrm{~d}, J=8.8 \mathrm{~Hz}, 2 \mathrm{H}) ;{ }^{13} \mathrm{CNMR}(75 \mathrm{MHz}$, $\left.\mathrm{CDCl}_{3}\right) \delta(\mathrm{ppm}) 14.0,22.6,25.9,28.9,29.1,29.3,31.7,67.7,103.9,110.1,114.9,116.2,124.6,129.8$, 130.8, 134.7, 153.2, 158.5; MS (EI) m/z $542\left(\mathrm{M}^{+}\right)$; HRESI-MS(positive ion) $\mathrm{C}_{36} \mathrm{H}_{47} \mathrm{O}_{4}\left([\mathrm{M}+\mathrm{H}]^{+}\right)$ requires 543.3474, found 543.3469. IR (KBr) v 3475, 3061, 2927, 2854, 1620, 1513, 1455, 1271, 1028 $\mathrm{cm}^{-1}$. Anal. Calcd. for $\mathrm{C}_{36} \mathrm{H}_{46} \mathrm{O}_{4}$ : C, 79.67; H, 8.54. Found: C, 79.65; H, 8.50.

7f, $R_{\mathrm{f}}=0.24$ (ethyl acetate/petroleum $\left.=1: 10\right)$, yield $\square 85 \%$, colourless sticky oil, $[\alpha] \mathrm{D}^{20}=-150.0^{\circ}(\mathrm{c}=$ 0.414, $\left.\mathrm{CHCl}_{3}\right) .{ }^{1} \mathrm{HNMR}\left(300 \mathrm{MHz}, \mathrm{CDCl}_{3}\right) \delta(\mathrm{ppm}) 0.88(\mathrm{tr}, J=6.9 \mathrm{~Hz}, 6 \mathrm{H}), 1.23-1.30(\mathrm{~m}, 36 \mathrm{H})$, 1.59-1.64 (m, 4H), 3.65-3.76 (m, 4H), $5.05(\mathrm{~s}, 2 \mathrm{H}), 6.48(\mathrm{~d}, J=2.4 \mathrm{~Hz}, 2 \mathrm{H}), 7.03$ (dd, J= 8.9 Hz, 2.4 Hz, $2 \mathrm{H}), 7.21(\mathrm{~d}, J=8.9 \mathrm{~Hz}, 2 \mathrm{H}), 7.78(\mathrm{~d}, J=8.9 \mathrm{~Hz}, 2 \mathrm{H}), 7.87(\mathrm{~d}, J=8.9 \mathrm{~Hz}, 2 \mathrm{H}) ;{ }^{13} \mathrm{CNMR}(75 \mathrm{MHz}$, $\left.\mathrm{CDCl}_{3}\right) \delta(\mathrm{ppm}) 14.10,22.67,25.94,28.95,29.33,29.50,29.53,29.61,29.62,31.90,67.78,103.91$, $109.95,114.93,116.33,124.65,129.87,131.03,134.71,153.22,158.56 ; \mathrm{MS}(\mathrm{EI}) \mathrm{m} / \mathrm{z} 654\left(\mathrm{M}^{+}\right)$; HRESI-MS(positive ion) $\mathrm{C}_{44} \mathrm{H}_{63} \mathrm{O}_{4}\left([\mathrm{M}+\mathrm{H}]^{+}\right.$) requires 655.4726, found 655.4721. IR ( $\left.\mathrm{KBr}\right)$ v 3529, 3061, 2924, 2853, 1621, 1513, 1456, 1271, $1028 \mathrm{~cm}^{-1}$. Anal. Calcd. for $\mathrm{C}_{44} \mathrm{H}_{52} \mathrm{O}_{4}$ : C, 80.69; H, 9.54. Found: C, 80.41; H, 9.27.

\section{General procedure for the asymmetric addition of phenylacetylene to aromatic aldehyde ${ }^{1}$}

Under nitrogen, phenylacetylene $(1.1 \mathrm{mmol}, 121 \mu \mathrm{L})$ and diethylzinc $(1.0 \mathrm{mmol}, 1.0 \mathrm{~mL}, 1 \mathrm{M}$ in hexane) were added to a flask $(25 \mathrm{~mL})$ containing toluene $(2.0 \mathrm{~mL})$. This solution was refluxed for $5 \mathrm{~h}$ and a white precipitate appeared. To the above reaction mixture was added 7,7'-disubsituted-BINOLs (>99\% ee, $0.10 \mathrm{mmol})$ and $\mathrm{CH}_{2} \mathrm{Cl}_{2}(8 \mathrm{~mL})$. After the mixture was stirred at room temperature for $15 \mathrm{~min}$, $\mathrm{Ti}\left(\mathrm{O}^{\mathrm{i}} \mathrm{Pr}\right)_{4}(0.25 \mathrm{mmol}, 74 \mu \mathrm{L})$ was added. After the reaction solution was stirred for another hour, 
aromatic aldehyde $(0.5 \mathrm{mmol})$ was then added, and the reaction mixture was stirred for $4 \mathrm{~h}$. The reaction was quenched with aqueous ammonium chloride. The organic layer was separated. The aqueous layer was extracted with ethyl acetate $(15 \mathrm{~mL} \times 3)$. The combined organic layer was washed with brine and dried over anhydrous $\mathrm{Na}_{2} \mathrm{SO}_{4}$. After removal of the solvent, the crude product was purified by chromatography on silical gel (ethyl acetate: petroleum $=1: 10$ ) to give the optically active propargylic alcohols.

\section{General procedure for the asymmetric addition of phenylacetylene to aliphatic aldehyde ${ }^{1 b}$}

A flask $(25 \mathrm{~mL})$ was charged with solution of phenylacetylene $(2.0 \mathrm{mmol}, 220 \mu \mathrm{L})$ and diethyl zinc $(2.0$ mmol, $2 \mathrm{~mL}, 1 \mathrm{M}$ in hexane) in tolunene $(1 \mathrm{~mL})$. After refluxed for 1 hour under nitrogen, the solution was cooled down to room temperature, and then 7,7'-dialloxyl-BINOL (>99\% ee, $80 \mathrm{mg}, 0.2 \mathrm{mmol}$ ), diethyl ether $(8 \mathrm{~mL})$ and $\mathrm{Ti}\left(\mathrm{O}^{\mathrm{i}} \mathrm{Pr}\right)_{4}(0.5 \mathrm{mmol}, 150 \mu \mathrm{L})$ were added sequentially. The solution was stirred for another hour, and aliphatic aldehyde $(0.5 \mathrm{mmol})$ was added. After stirred for additional $4 \mathrm{~h}$, the reaction was quenched with saturated ammonium chloride, the resulting mixture was extracted with ethyl acetate $(15 \mathrm{~mL} \times 3)$. The combined organic layer was washed with brine and dried over anhydrous $\mathrm{Na}_{2} \mathrm{SO}_{4}$. Purification of the crude product by column chromatography on silical gel (ethyl acetate: petroleum $=1: 10$ ) afforded the product. ${ }^{1}$

1,3-Diphenyl-prop-2-yn-1-ol: 91\% yield, 95\% ee determined by HPLC analysis (Chiralcel OD column, $10 \%$ IPA in hexane, rate: $1 \mathrm{~mL} / \mathrm{min}, 254 \mathrm{~nm})$. Retention time: $\mathrm{t}_{\mathrm{R}}($ minor $)=12.20$ and $\mathrm{t}_{\mathrm{R}}($ major $)=20.76$ $\min .[\alpha]^{20}{ }_{\mathrm{D}}=-9.1^{\mathrm{o}}\left(\mathrm{c}=1.68, \mathrm{CHCl}_{3}\right) .{ }^{1} \mathrm{HNMR}\left(300 \mathrm{MHz}, \mathrm{CDCl}_{3}\right) \delta(\mathrm{ppm}) 2.21(\mathrm{~d}, J=6.0 \mathrm{~Hz}, 1 \mathrm{H}), 5.63$ $(\mathrm{d}, J=6.0 \mathrm{~Hz}, 1 \mathrm{H}), 7.24-7.35(\mathrm{~m}, 6 \mathrm{H}), 7.40-7.43(\mathrm{~m}, 2 \mathrm{H}), 7.55(\mathrm{~m}, 2 \mathrm{H})$.

1-(4-Methylphenyl)-3-phenyl-prop-2-yn-1-ol: 71\% yield, 92\% ee determined by HPLC analysis (Chiralcel OD column, 10\% IPA in hexane, rate: $1 \mathrm{~mL} / \mathrm{min}, 254 \mathrm{~nm}$ ). Retention time: $\mathrm{t}_{\mathrm{R}}($ minor) $=9.14$ and $\mathrm{t}_{\mathrm{R}}($ major $)=18.71 \mathrm{~min} .[\alpha]^{20}{ }_{\mathrm{D}}=-3.6^{\mathrm{o}}\left(\mathrm{c}=1.17, \mathrm{CHCl}_{3}\right),{ }^{1} \mathrm{HNMR}\left(300 \mathrm{MHz}, \mathrm{CDCl}_{3}\right) \delta(\mathrm{ppm}) 2.26(\mathrm{~s}$, 1H), $2.29(\mathrm{~s}, 3 \mathrm{H}), 5.58(\mathrm{~s}, 1 \mathrm{H}), 7.12-7.18(\mathrm{~m}, 2 \mathrm{H}), 7.23-7.26(\mathrm{~m}, 3 \mathrm{H}), 7.38-7.44(\mathrm{~m}, 4 \mathrm{H})$.

1-(1-Naphthyl)-3-phenyl-prop-2-yn-1-ol: 83\% yield, 94\% ee determined by HPLC analysis (Chiralcel OD column, $15 \%$ IPA in hexane, rate: $1 \mathrm{~mL} / \mathrm{min}, 254 \mathrm{~nm})$. Retention time: $\mathrm{t}_{\mathrm{R}}($ minor $)=10.14$ and $\mathrm{t}_{\mathrm{R}}($ major $)=18.68 \min .[\alpha]_{\mathrm{D}}^{20}=+24.1^{\mathrm{o}}\left(\mathrm{c}=2.56, \mathrm{CHCl}_{3}\right),{ }^{1} \mathrm{HNMR}\left(300 \mathrm{MHz}, \mathrm{CDCl}_{3}\right) \delta(\mathrm{ppm}) 2.40(\mathrm{~s}$, 1H), $6.28(\mathrm{~s}, 1 \mathrm{H}), 7.23-7.26(\mathrm{~m}, 3 \mathrm{H}), 7.40-7.44(\mathrm{~m}, 5 \mathrm{H}), 7.51-7.84(\mathrm{~m}, 3 \mathrm{H}), 8.31(\mathrm{~d}, \mathrm{~J}=8.4 \mathrm{~Hz}, 1 \mathrm{H})$.

1-piperonyl-3-phenyl-prop-2-yn-1-ol: 87\% yield, 92\% ee determined by HPLC analysis (Chiralcel OD column, 15\% IPA in hexane, rate: $1 \mathrm{~mL} / \mathrm{min}, 254 \mathrm{~nm}$ ). Retention time: $\mathrm{t}_{\mathrm{R}}($ minor $)=9.44$ and $\mathrm{t}_{\mathrm{R}}($ major $)=20.07 \min .[\alpha]_{\mathrm{D}}^{20}=-33.1^{\mathrm{o}}\left(\mathrm{c}=1.42, \mathrm{CHCl}_{3}\right),{ }^{1} \mathrm{HNMR}\left(300 \mathrm{MHz}, \mathrm{CDCl}_{3}\right) \delta(\mathrm{ppm}) 2.30(\mathrm{~s}$, 
1H), 5.59 (s, 1H), $5.98(\mathrm{~s}, 2 \mathrm{H}), 6.82(\mathrm{~d}, J=8.0 \mathrm{~Hz}, 1 \mathrm{H}), 7.07-7.13(\mathrm{~m}, 2 \mathrm{H}), 7.30-7.33$ (m, 3H), 7.46-7.49 $(\mathrm{m}, 2 \mathrm{H})$.

1-(4-chlorophenyl)-3-phenyl-prop-2-yn-1-ol: 79\% yield, 93\% ee determined by HPLC analysis (Chiralcel OD column, 10\% IPA in hexane, rate: $1 \mathrm{~mL} / \mathrm{min}, 254 \mathrm{~nm}$ ). Retention time: $\mathrm{t}_{\mathrm{R}}($ minor) $=8.69$ and $\mathrm{t}_{\mathrm{R}}$ (major) $=28.84 \mathrm{~min} .[\alpha]^{20}{ }_{\mathrm{D}}=-8.8^{\mathrm{o}}\left(\mathrm{c}=0.672, \mathrm{CHCl}_{3}\right),{ }^{1} \mathrm{HNMR}\left(300 \mathrm{MHz}, \mathrm{CDCl}_{3}\right) \delta(\mathrm{ppm}) 2.38(\mathrm{~d}$, $J=6.0 \mathrm{~Hz}, 1 \mathrm{H}), 5.67(\mathrm{~d}, J=6.0 \mathrm{~Hz}, 1 \mathrm{H}), 7.32-7.39(\mathrm{~m}, 5 \mathrm{H}), 7.45-7.49$ (m, 2H), 7.54-7.57 (m, 2H).

1,5-Diphenyl-pent-1-en-4-yne-3-ol: 69\% yield, 93\% eе determined by HPLC analysis (Chiralcel OD column, 20\% IPA in hexane, rate: $1 \mathrm{~mL} / \mathrm{min}, 254 \mathrm{~nm})$. Retention time: $\mathrm{t}_{\mathrm{R}}($ minor $)=9.19$ and $\mathrm{t}_{\mathrm{R}}($ major $)=$ 21.27 min. $[\alpha]^{20}{ }_{\mathrm{D}}=-1.9^{\circ}\left(\mathrm{c}=0.984, \mathrm{CHCl}_{3}\right),{ }^{1} \mathrm{HNMR}\left(300 \mathrm{MHz}, \mathrm{CDCl}_{3}\right) \delta(\mathrm{ppm}) 2.12(\mathrm{~s}, 1 \mathrm{H}), 5.24(\mathrm{~s}$, 1H), 6.33 (dd, $J=16.0 \mathrm{~Hz}, 7.5 \mathrm{~Hz}, 1 \mathrm{H}), 6.79$ (d, $J=16.0 \mathrm{~Hz}, 1 \mathrm{H}), 6.77-7.44(\mathrm{~m}, 10 \mathrm{H})$.

1-(3-Methyl-phenyl)-3-phenyl-prop-2-yn-1-ol: 80\% yield, 92\% ee determined by HPLC analysis (Chiralcel OD column, 10\% IPA in hexane, rate: $1 \mathrm{~mL} / \mathrm{min}, 254 \mathrm{~nm}$ ). Retention time: $\mathrm{t}_{\mathrm{R}}($ minor) $=9.74$ and $\mathrm{t}_{\mathrm{R}}$ (major) $=23.59 \mathrm{~min} .[\alpha]^{20}{ }_{\mathrm{D}}=-6.5^{\mathrm{o}}\left(\mathrm{c}=2.52, \mathrm{CHCl}_{3}\right),{ }^{1} \mathrm{HNMR}\left(300 \mathrm{MHz}, \mathrm{CDCl}_{3}\right) \delta(\mathrm{ppm}) 2.46(\mathrm{~s}$, 3H), $2.54(\mathrm{~s}, 2 \mathrm{H}), 5.67(\mathrm{~s}, 1 \mathrm{H}), 7.19(\mathrm{~m}, 1 \mathrm{H}), 7.31-7.34(\mathrm{~m}, 4 \mathrm{H}), 7.44(\mathrm{~m}, 2 \mathrm{H}), 7.48-7.51(\mathrm{~m}, 2 \mathrm{H})$.

1-(4-Methoxy-phenyl)-3-phenyl-prop-2-yn-1-ol: 93\% yield, 91\% ee determined by HPLC analysis (Chiralcel OD column, 10\% IPA in hexane, rate: $1 \mathrm{~mL} / \mathrm{min}, 254 \mathrm{~nm}$ ). Retention time: $\mathrm{t}_{\mathrm{R}}($ minor)= 13.54 and $\mathrm{t}_{\mathrm{R}}$ (major) $=27.42 \mathrm{~min} .[\alpha]^{20}{ }_{\mathrm{D}}=-6.2^{\circ}\left(\mathrm{c}=1.45, \mathrm{CHCl}_{3}\right),{ }^{1} \mathrm{HNMR}\left(300 \mathrm{MHz}, \mathrm{CDCl}_{3}\right) \delta(\mathrm{ppm}) 2.23(\mathrm{~s}$, $1 \mathrm{H}), 3.76(\mathrm{~s}, 3 \mathrm{H}), 5.71(\mathrm{~s}, 1 \mathrm{H}), 6.84-6.87(\mathrm{~m}, 2 \mathrm{H}), 7.24-7.27(\mathrm{~m}, 3 \mathrm{H}), 7.39-7.42(\mathrm{~m}, 2 \mathrm{H})$, 7.46-7.49 (m, $2 \mathrm{H})$.

4-Methyl-1-phenyl-pent-1-yn-ol. 64\% yield, 92\% ee determined by HPLC analysis (Chiralcel OD column, 10\% IPA in hexane, rate: $1 \mathrm{~mL} / \mathrm{min}, 254 \mathrm{~nm})$. Retention time: $\mathrm{t}_{\mathrm{R}}($ minor $)=5.28$ and $\mathrm{t}_{\mathrm{R}}($ major $)=$ $9.52 \min .[\alpha]^{20}{ }_{\mathrm{D}}=+2.7^{\mathrm{o}}\left(\mathrm{c}=0.946, \mathrm{CHCl}_{3}\right),{ }^{1} \mathrm{HNMR}\left(300 \mathrm{MHz}, \mathrm{CDCl}_{3}\right) \delta(\mathrm{ppm}) 1.02(\operatorname{tr}, J=6.6 \mathrm{~Hz}$, $3 \mathrm{H}), 1.79(\mathrm{~s}, 1 \mathrm{H}), 1.87-1.95(\mathrm{~m}, 1 \mathrm{H}), 4.34(\mathrm{~m}, 1 \mathrm{H}), 7.23-7.28(\mathrm{~m}, 3 \mathrm{H})$, 7.35-7.40 (m, 2H).

1-phenyl-hept-1-yn-3-ol. 73\% yield, 93\% ee determined by HPLC analysis (Chiralcel OD column, $20 \%$ IPA in hexane, rate: $1 \mathrm{~mL} / \mathrm{min}, 254 \mathrm{~nm})$. Retention time: $\mathrm{t}_{\mathrm{R}}($ minor $)=5.60$ and $\mathrm{t}_{\mathrm{R}}($ major $)=14.37 \mathrm{~min}$. $[\alpha]^{20}{ }_{\mathrm{D}}=+1.5^{\mathrm{o}}\left(\mathrm{c}=1.11, \mathrm{CHCl}_{3}\right),{ }^{1} \mathrm{HNMR}\left(300 \mathrm{MHz}, \mathrm{CDCl}_{3}\right) \delta(\mathrm{ppm}) 0.88(\mathrm{tr}, J=7.2 \mathrm{~Hz}, 3 \mathrm{H}), 1.32-1.48$ (m, 4H), 1.72-1.77 (m, 2H), 2.11 (br, 1H), 4.54 (tr, $J=6.6 \mathrm{~Hz}, 1 \mathrm{H}), 7.20-7.27$ (m, 3H), 7.36-7.39 (m, 2H). References:

1. (a) Moore, D.; Pu, L. Org. Lett. 2002, 4, 1855. (b) Gao, G.; Moore, D.; Xie, R. G.; Pu, L. Org. Lett. 2002, 4, 4143. (c) Lu, G.; Li, X.; Chan, A. S. C. Chem. Commun. 2002, 172. (d) Li, X.; Lu, G.; 
Kwok, W. H.; Chan, A. S. C. J. Am. Chem. Soc. 2002, 124, 12636. 
${ }^{1}$ HNMR for compound 3

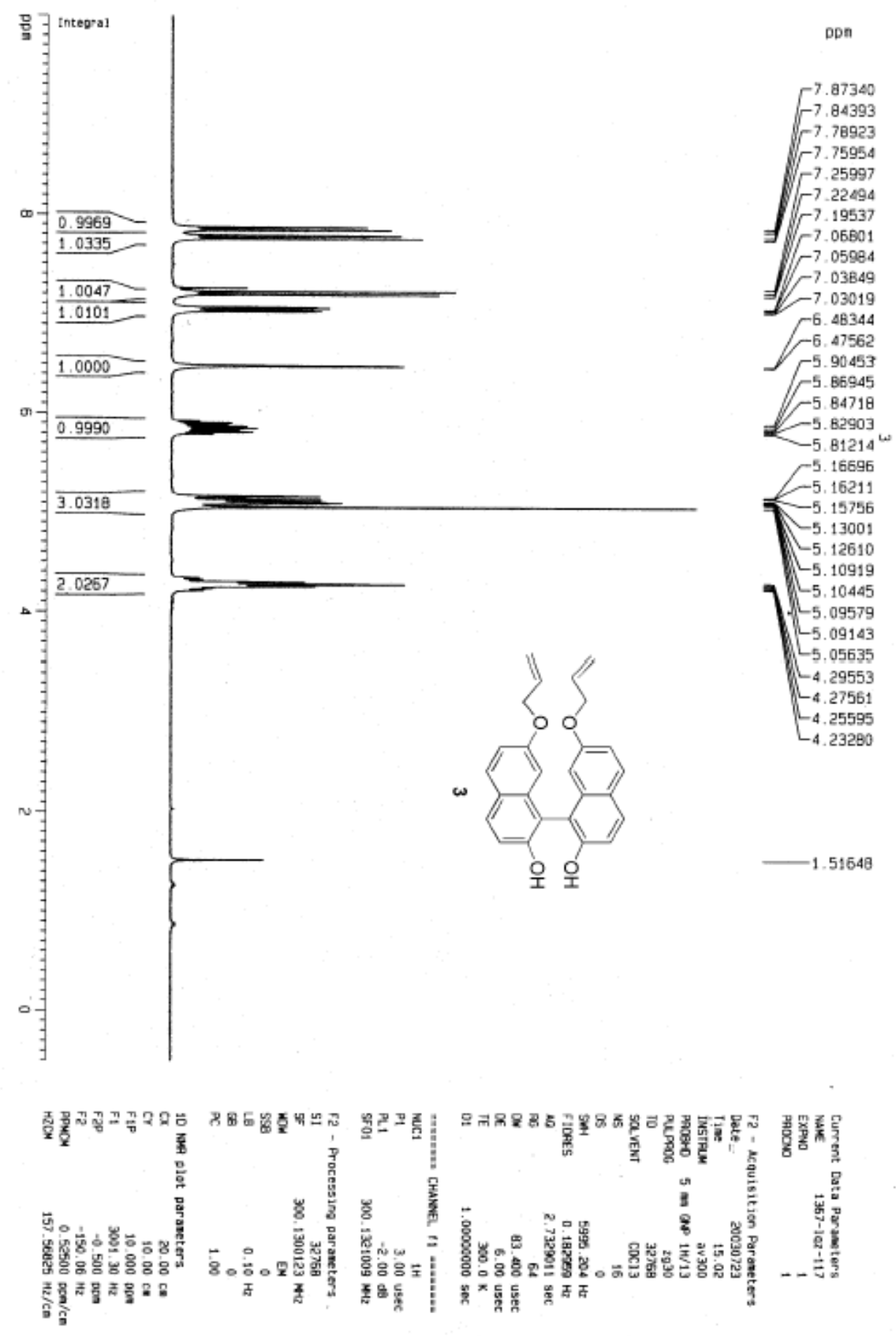


${ }^{13}$ CNMR for compound $\mathbf{3}$

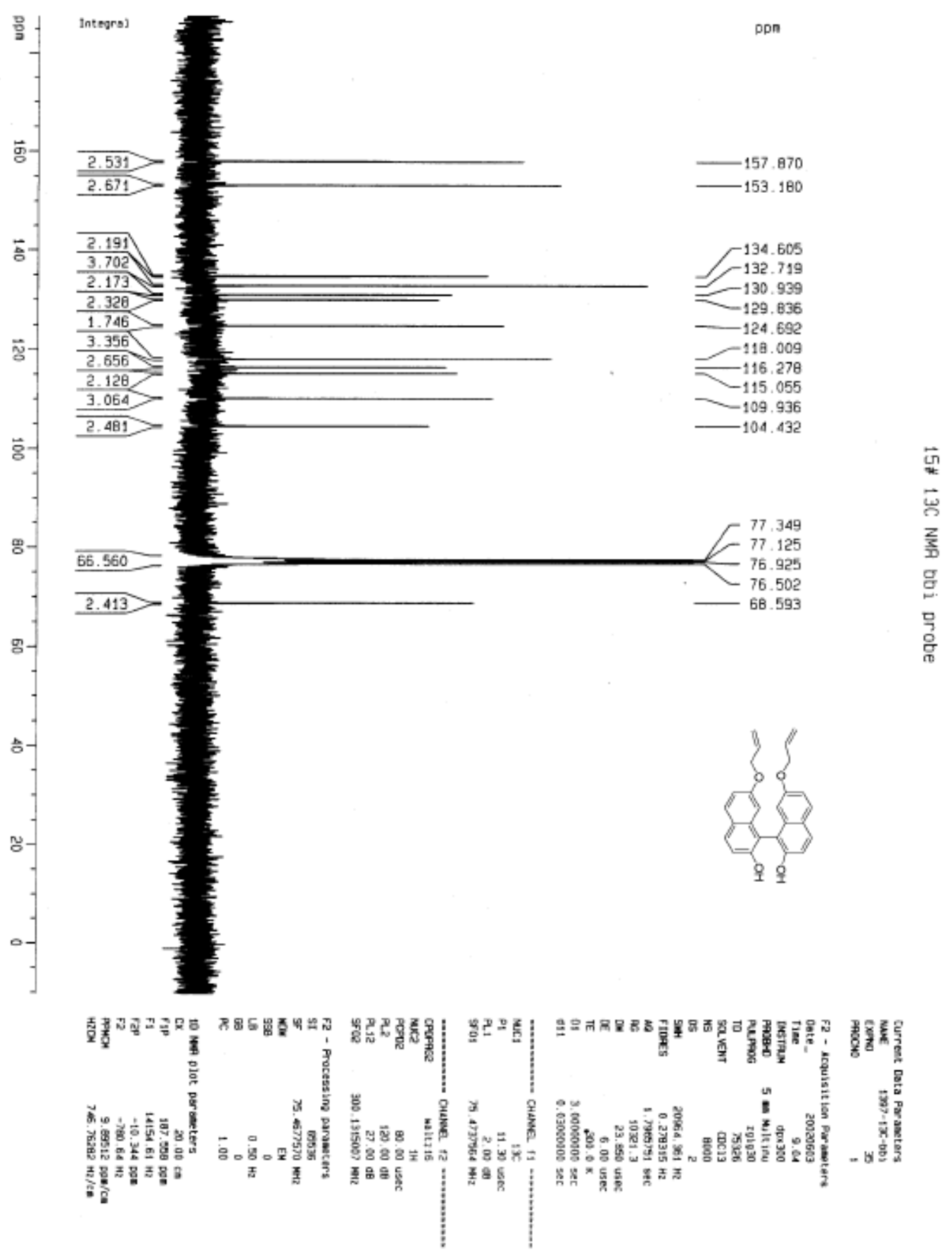


HPLC for racemic 3

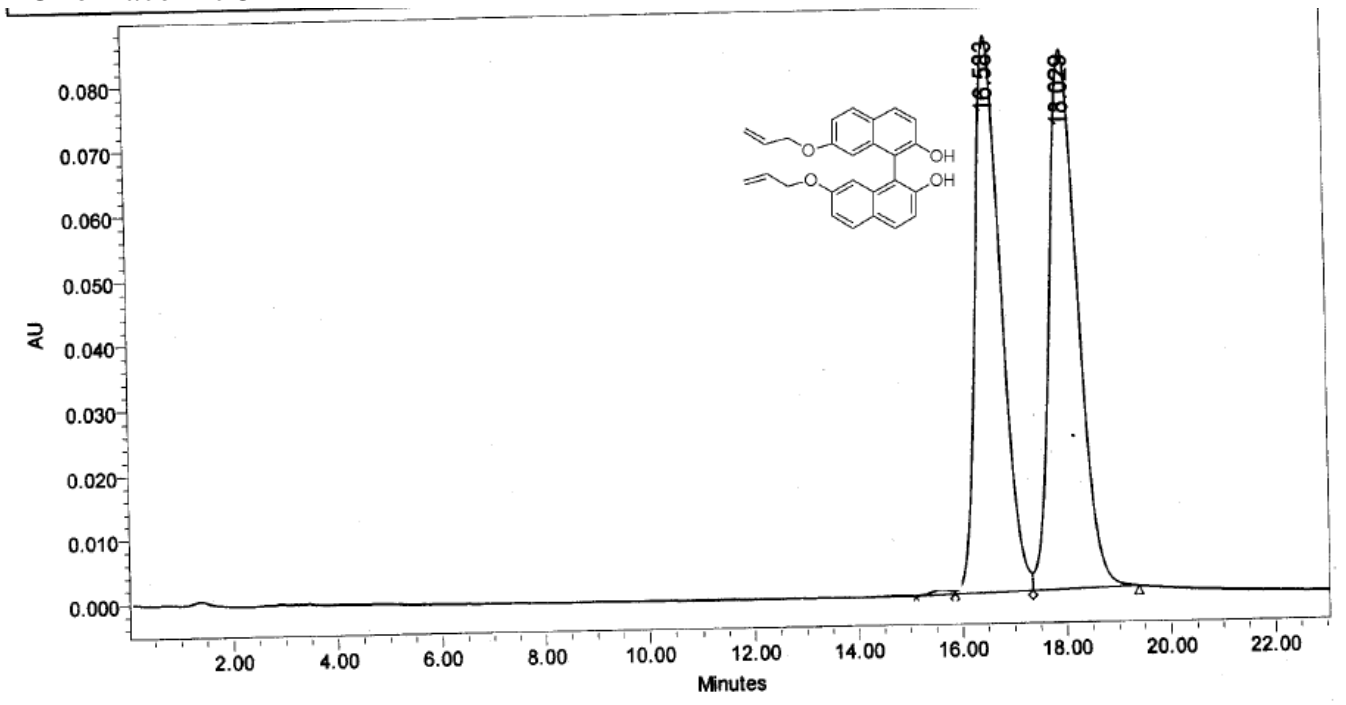

HPLC for Optically Pure 3

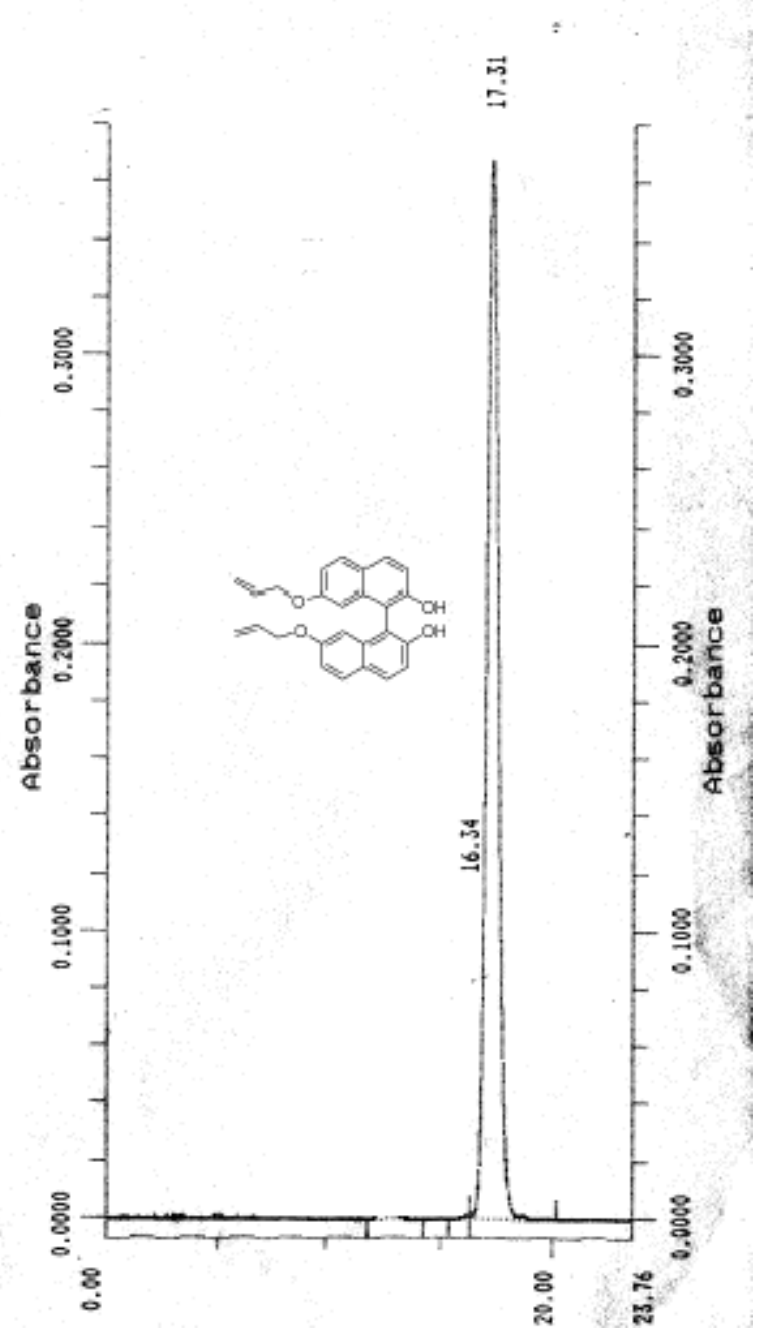


${ }^{1}$ HNMR for compound 5

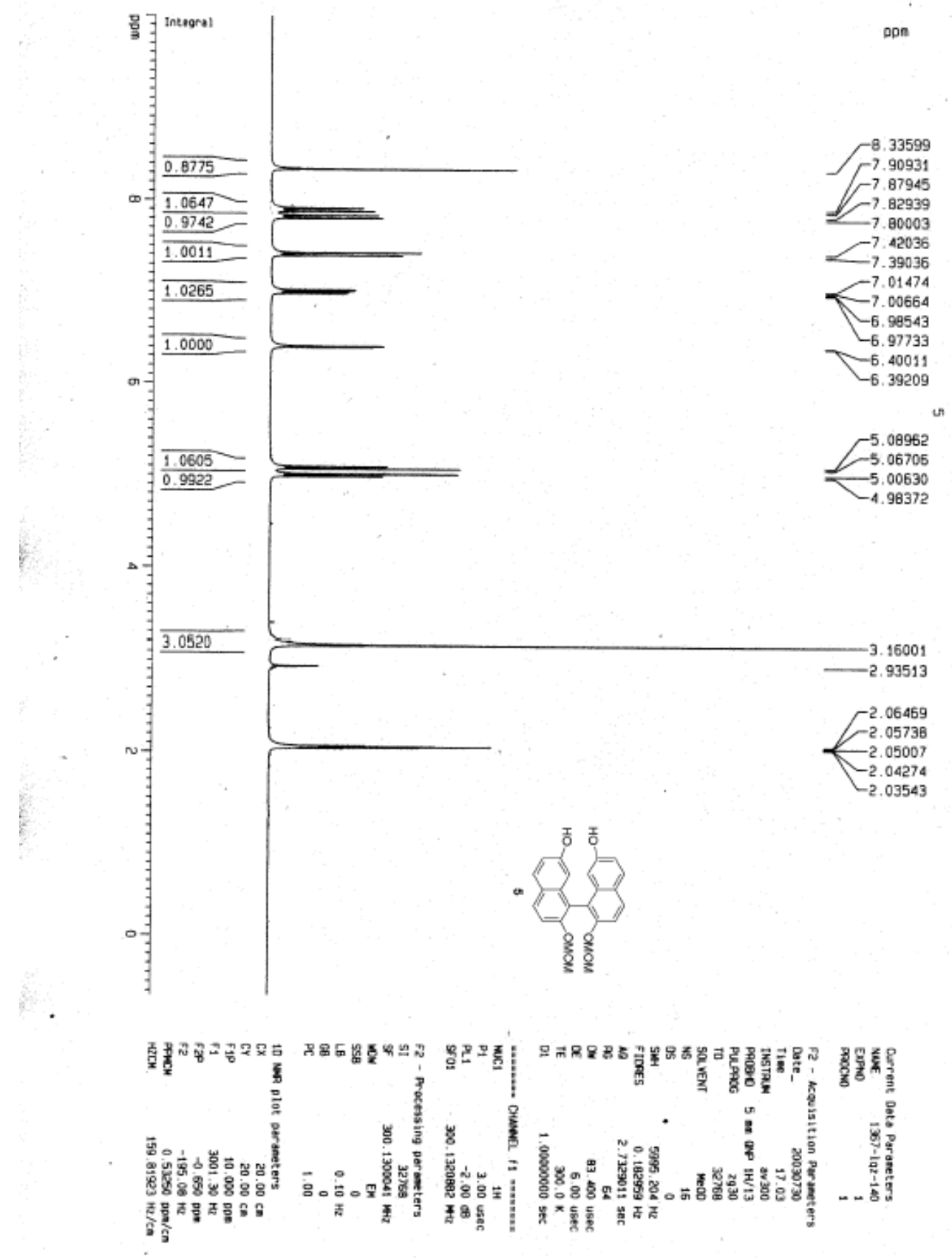


${ }^{13}$ CNMR for compound 5

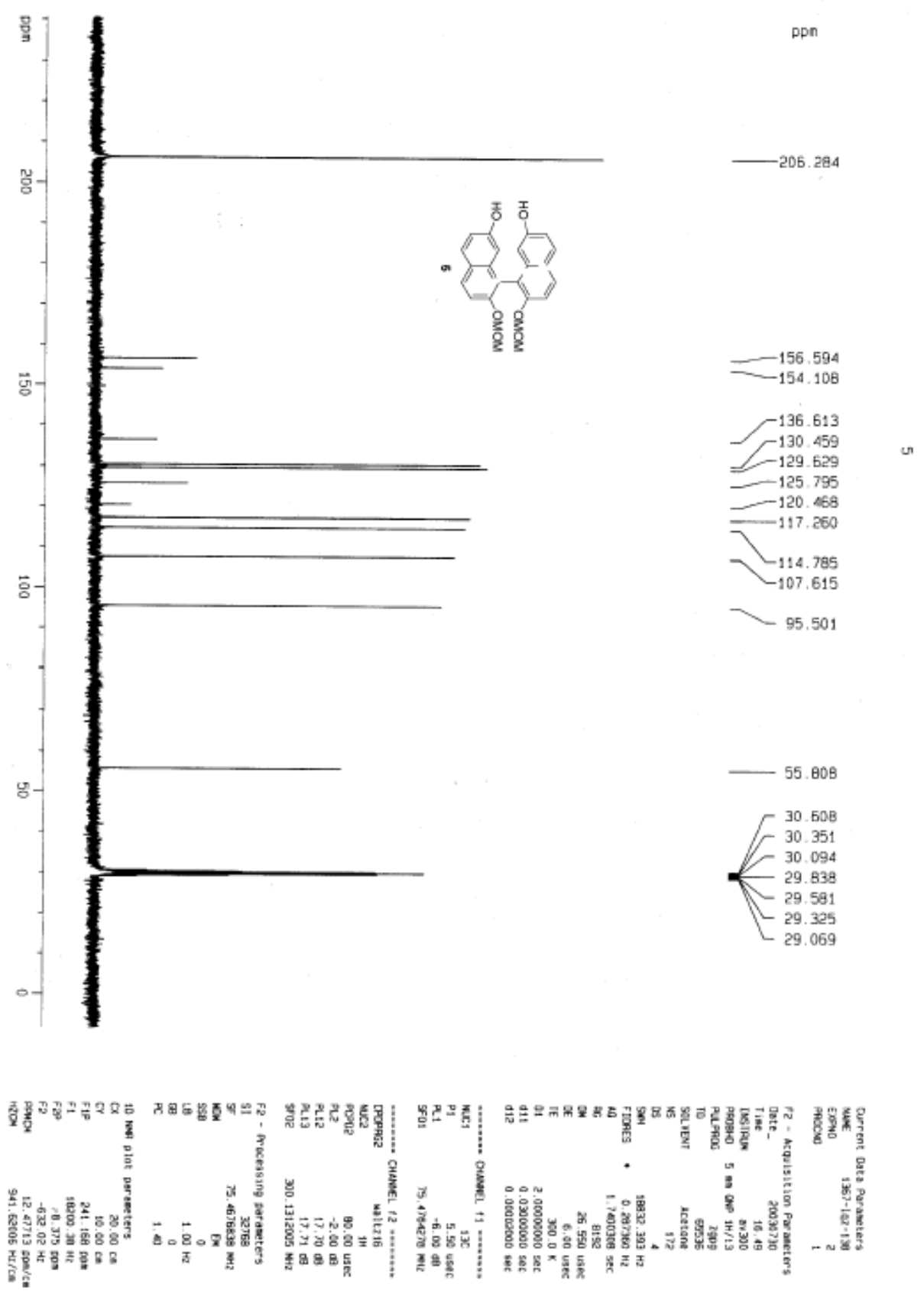


${ }^{1}$ HNMR for compound 7a

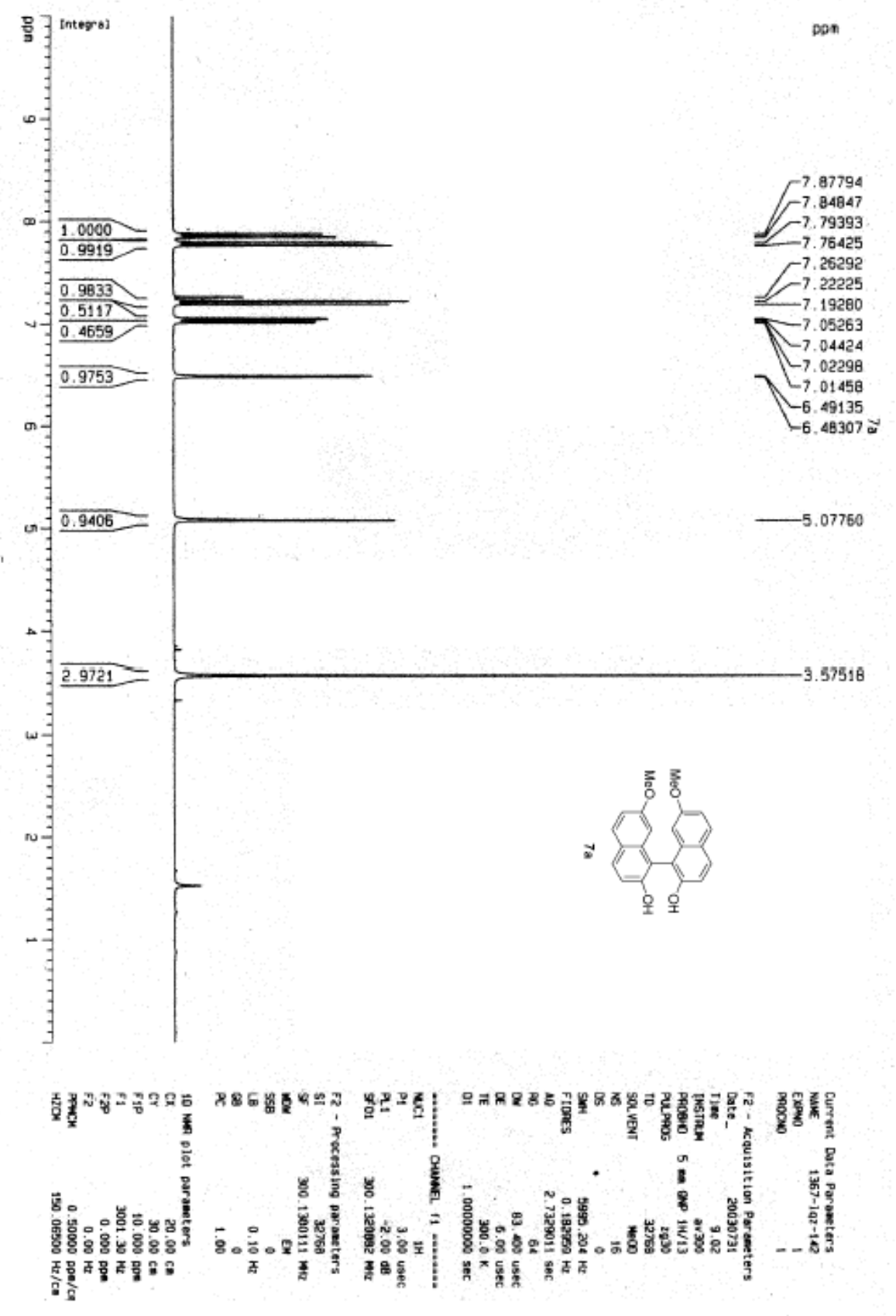


${ }^{13}$ CNMR for compound $7 a$

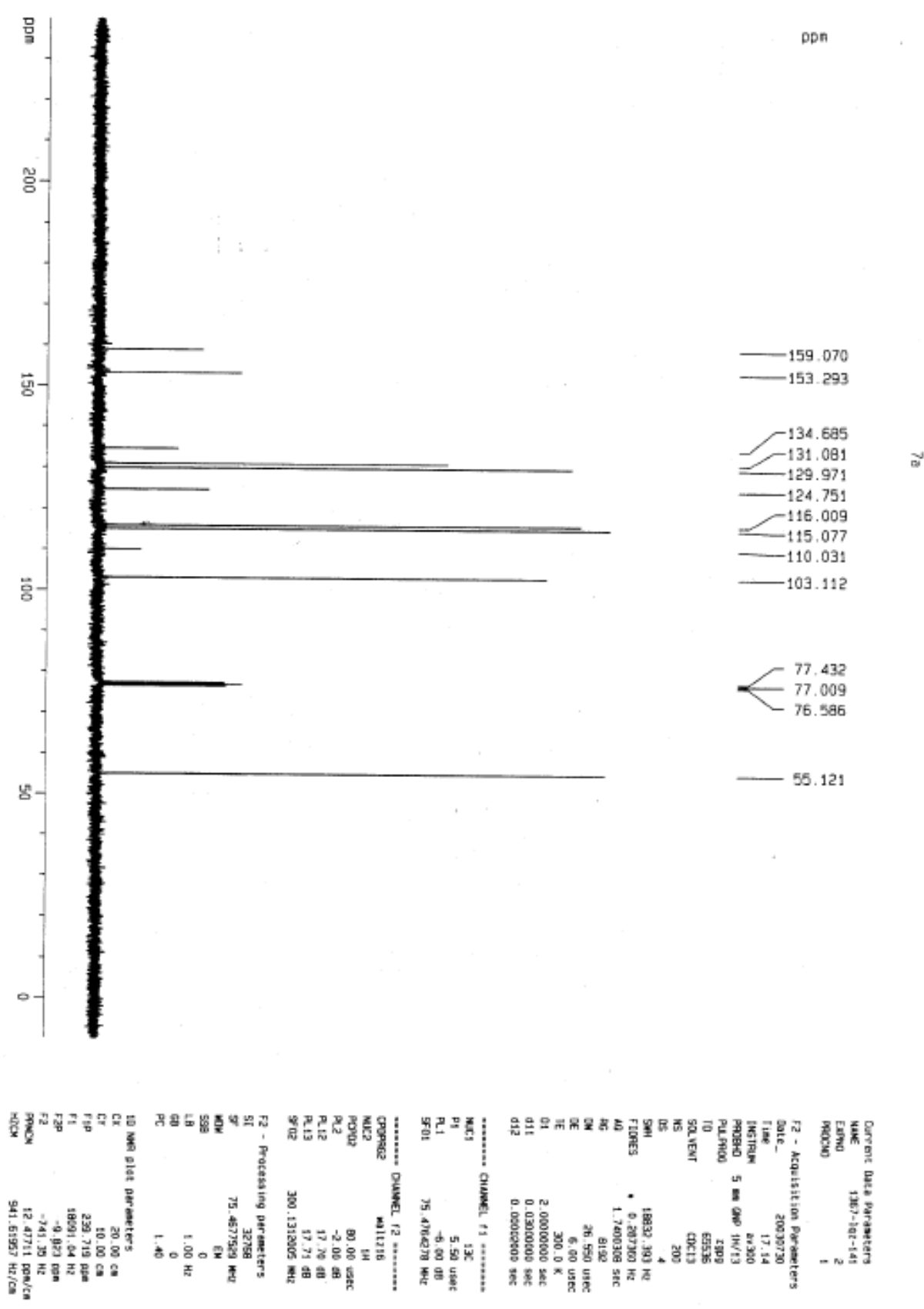


${ }^{1}$ HNMR for compound $\mathbf{7 b}$
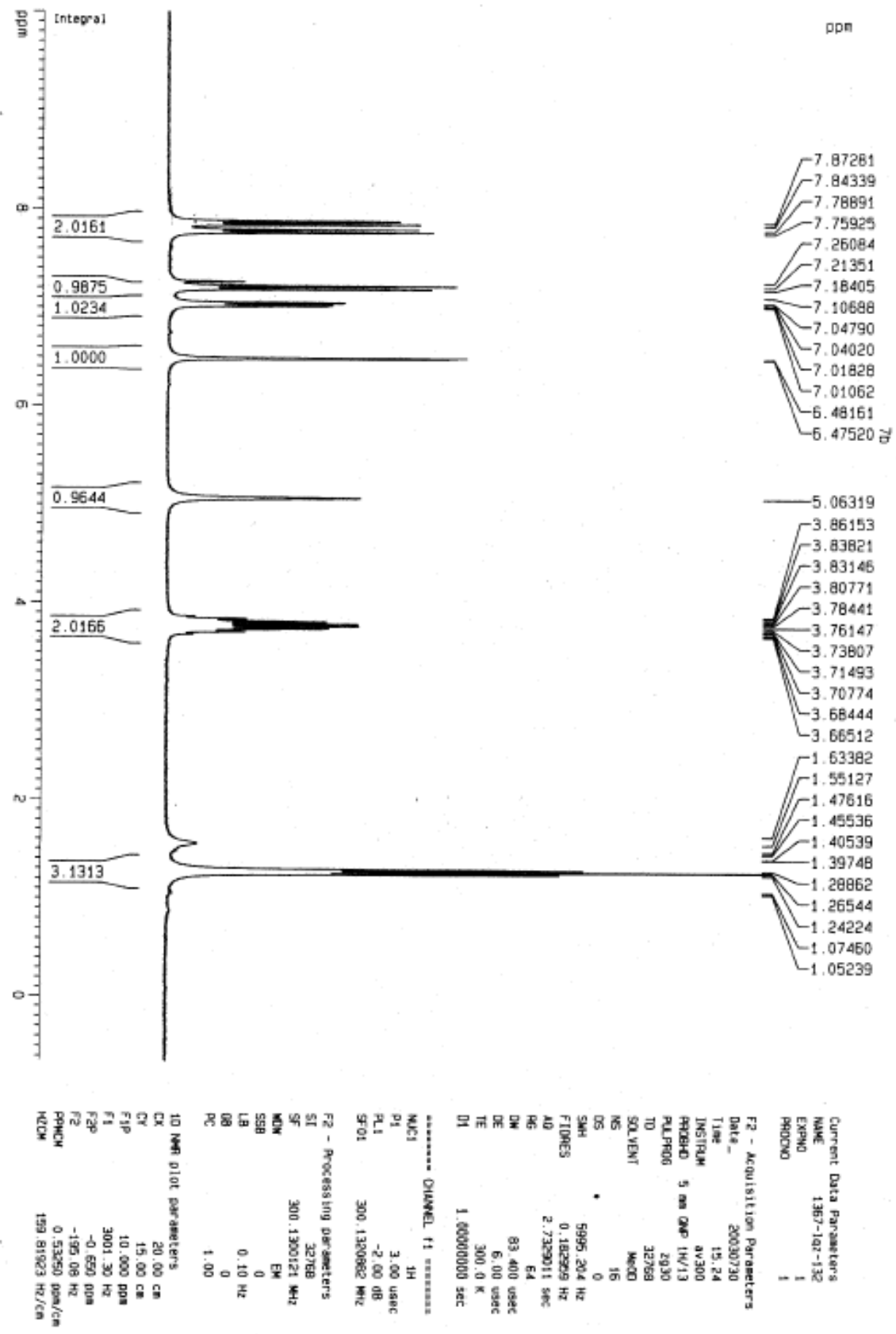
${ }^{13}$ CNMR for compound $7 \mathbf{b}$
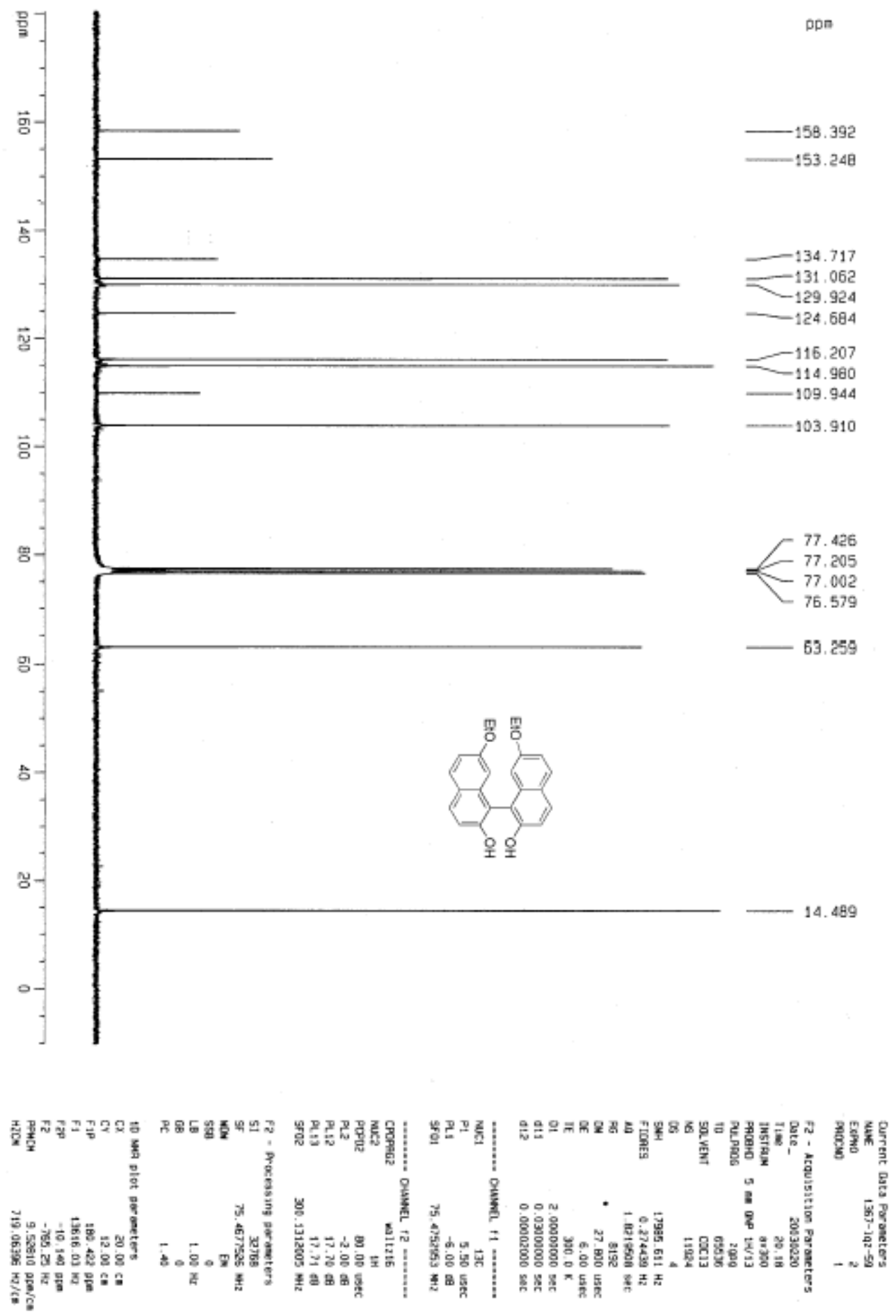
${ }^{1}$ HNMR for compound 7c
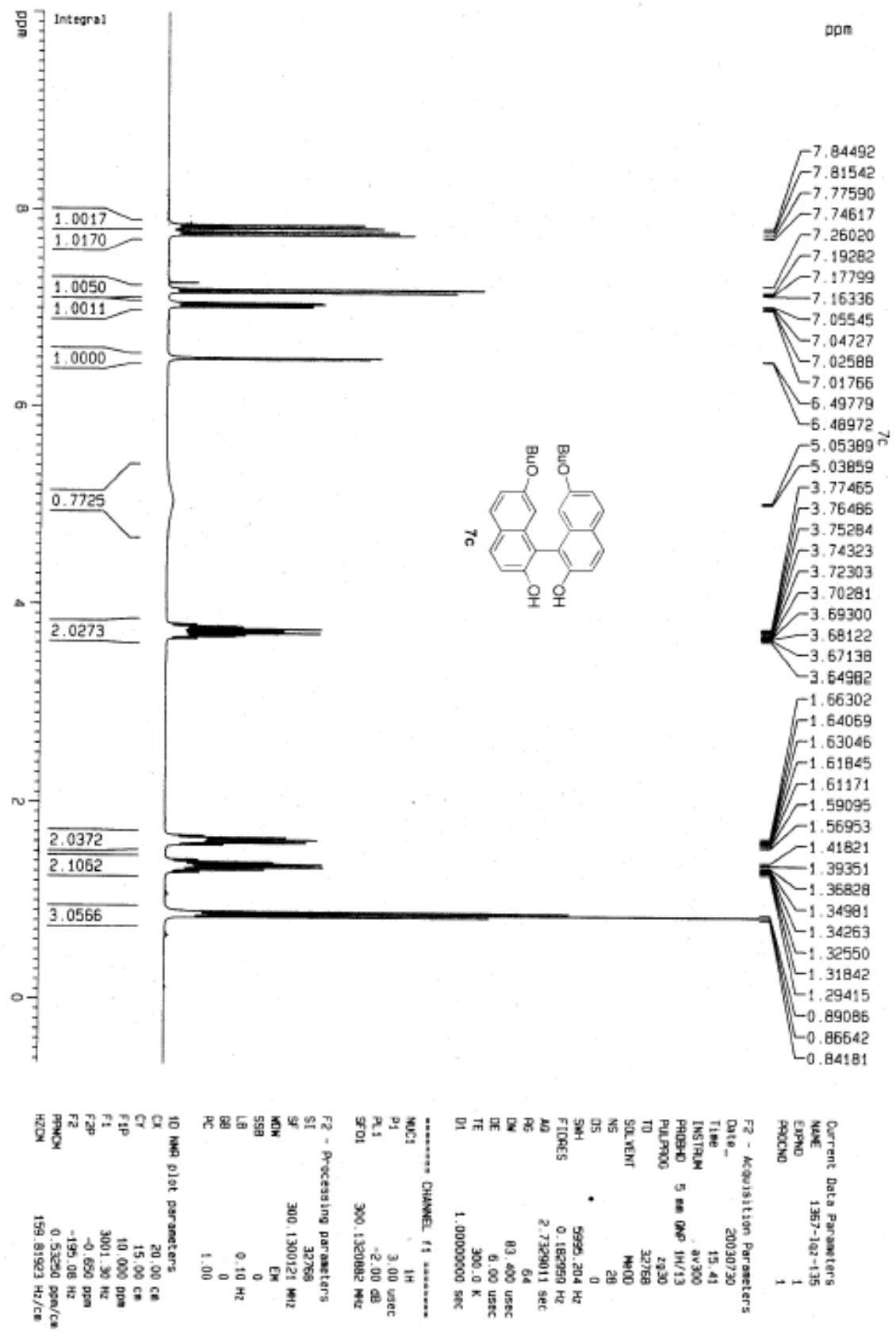
${ }^{13}$ CNMR for compound $7 \mathbf{c}$

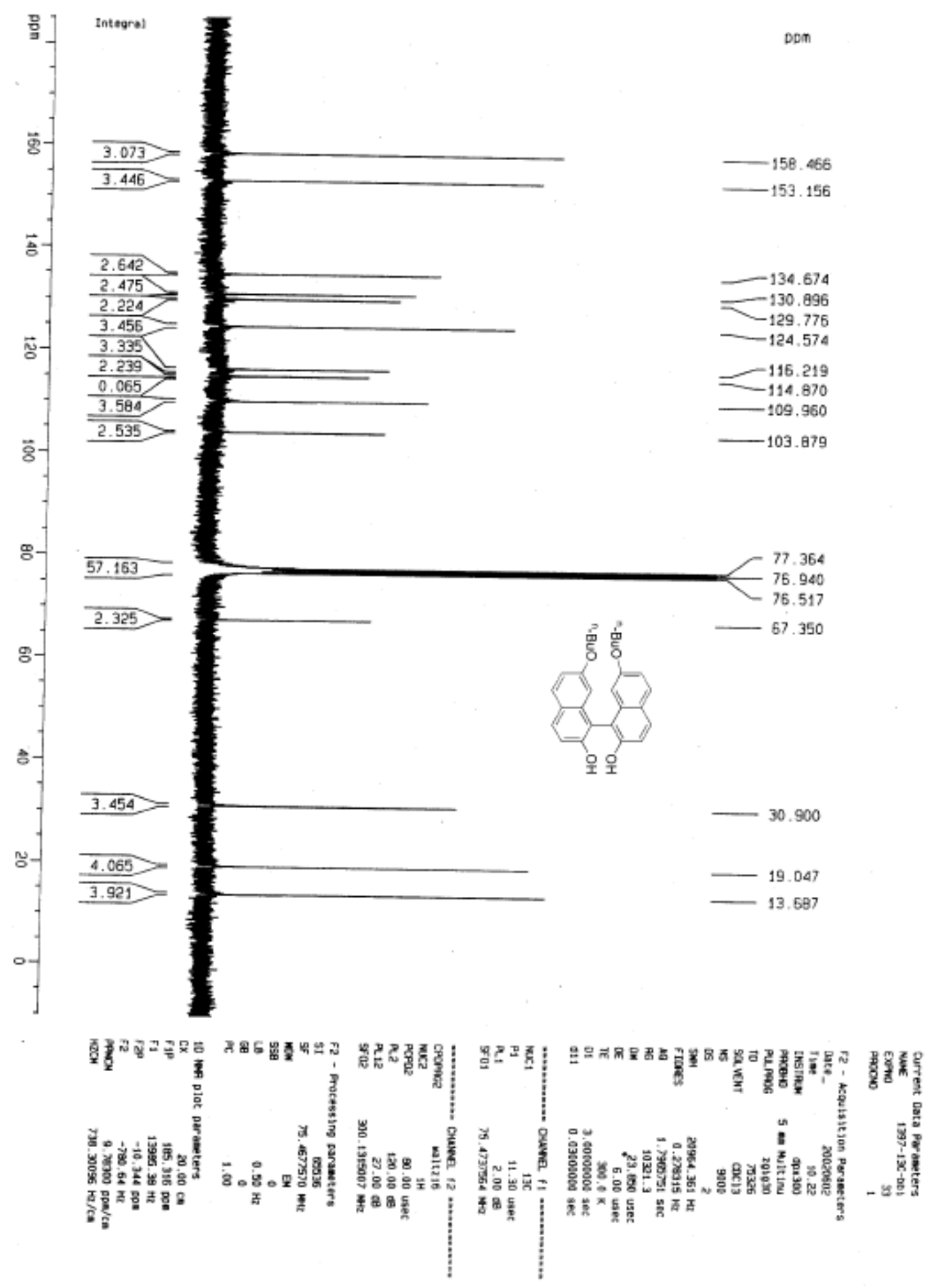


${ }^{1}$ HNMR for compound 7d

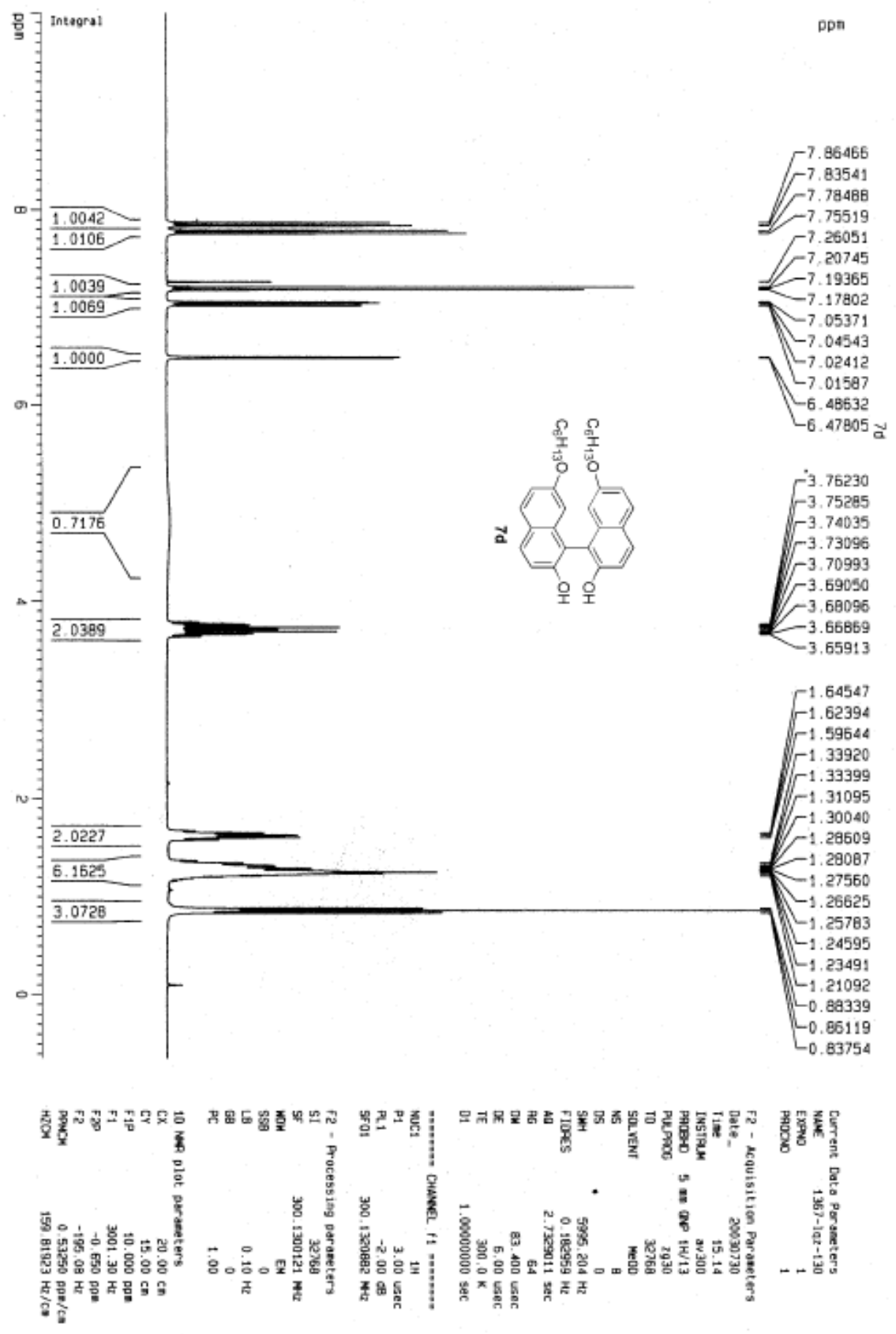


${ }^{13}$ CNMR for compound 7d

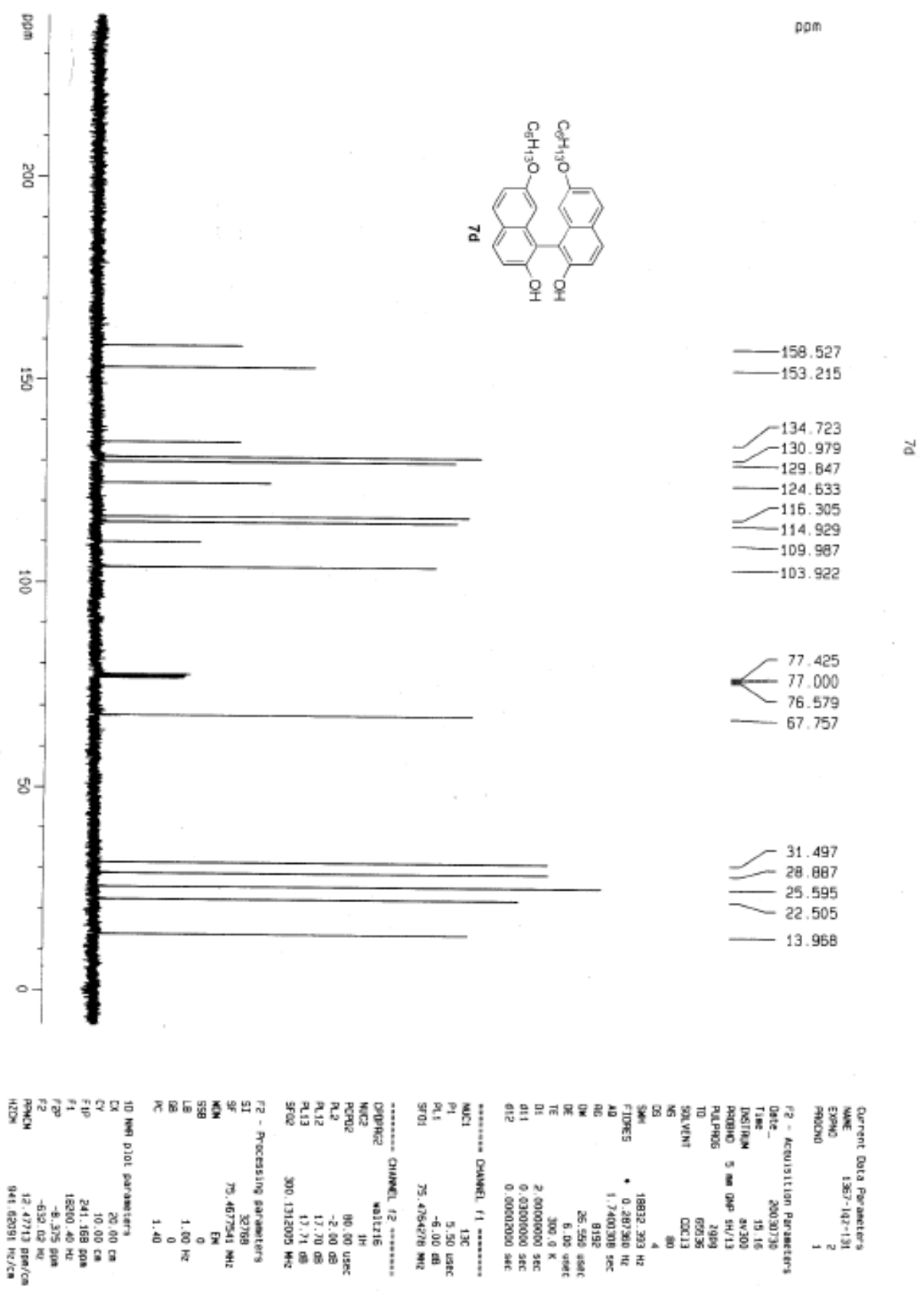


${ }^{1}$ HNMR for compound 7e

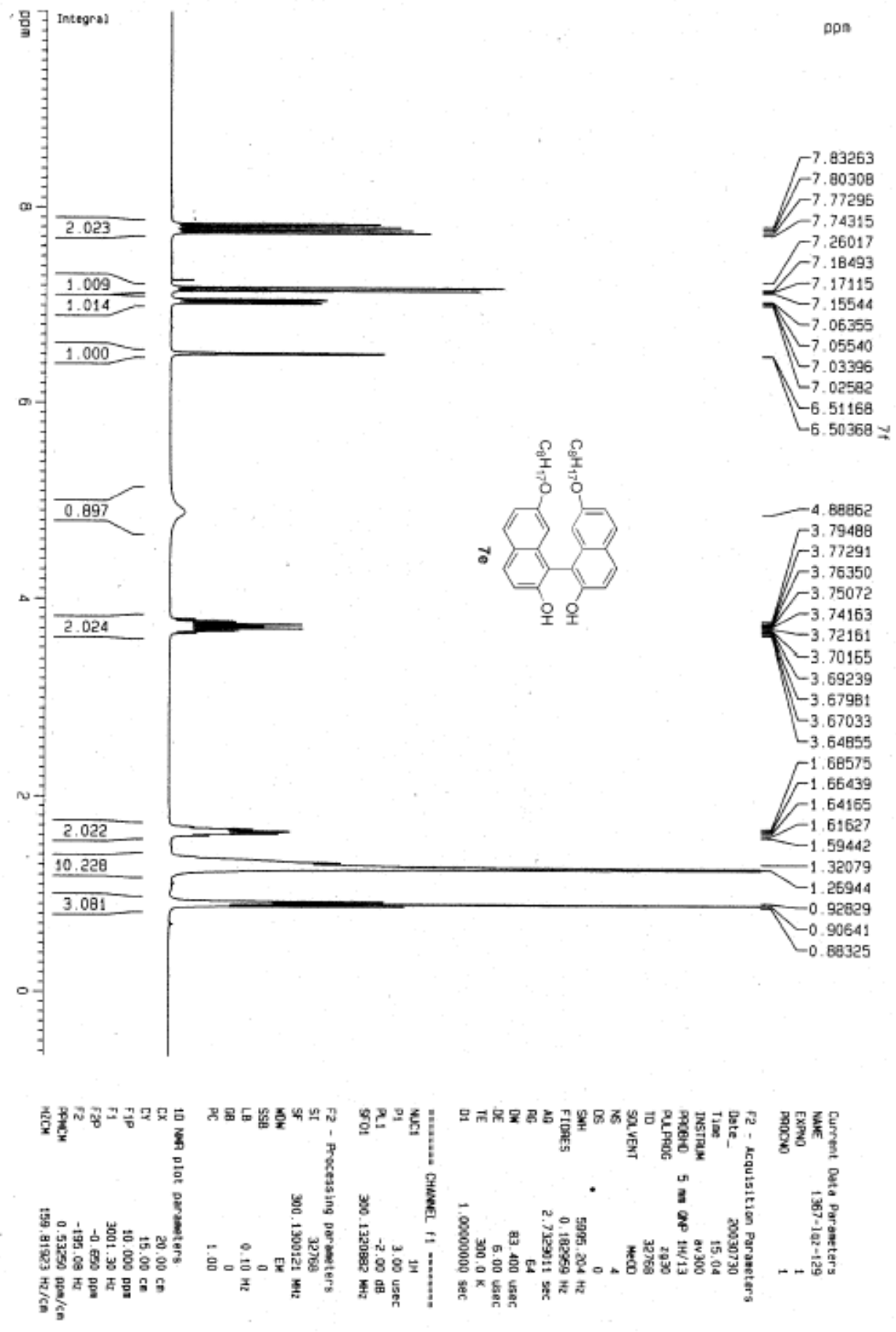


${ }^{13}$ CNMR for compound 7e

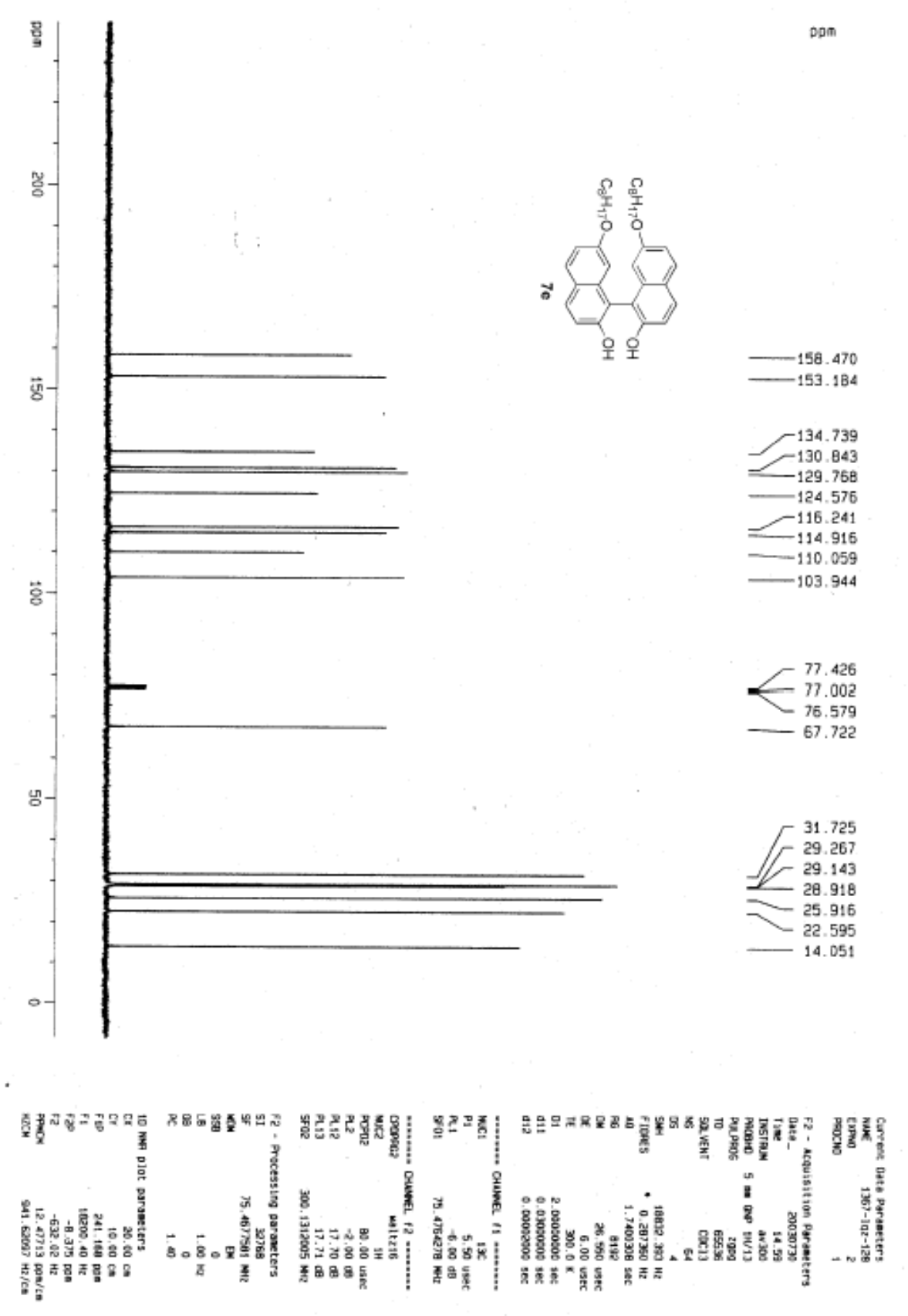


${ }^{1}$ HNMR for compound $7 \mathbf{f}$
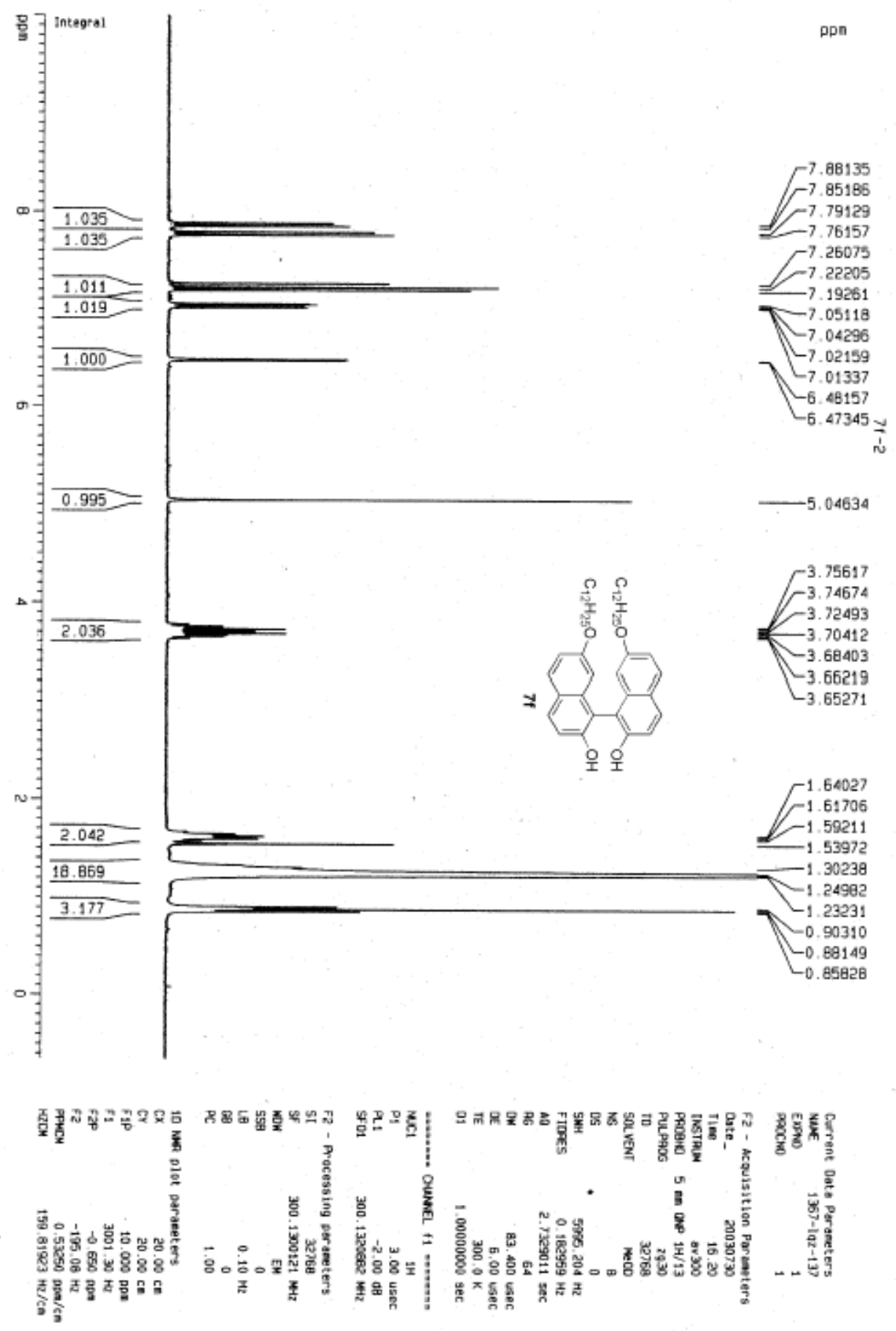
${ }^{13}$ CNMR for compound $7 f$

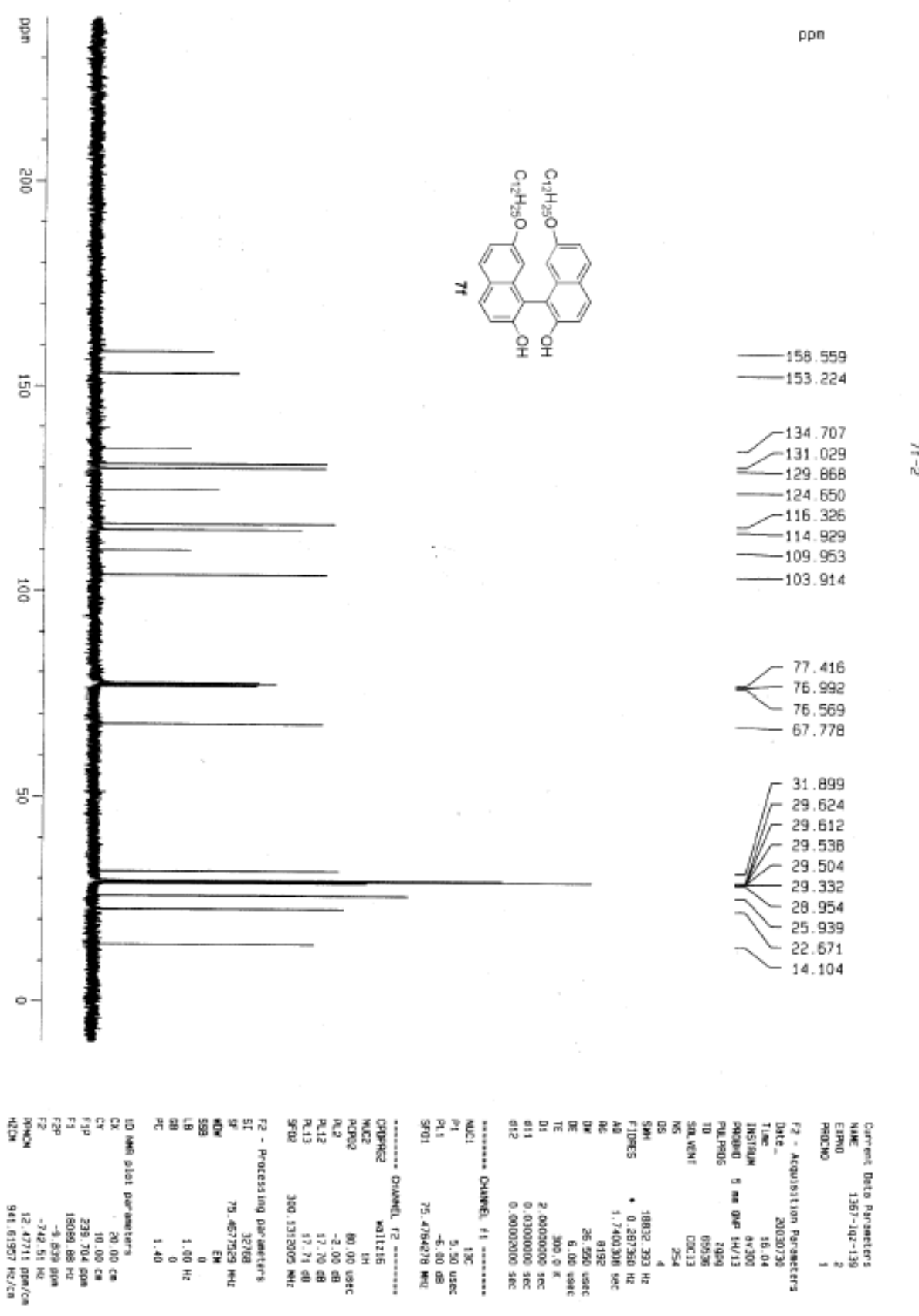


${ }^{1}$ HNMR for compound 8a
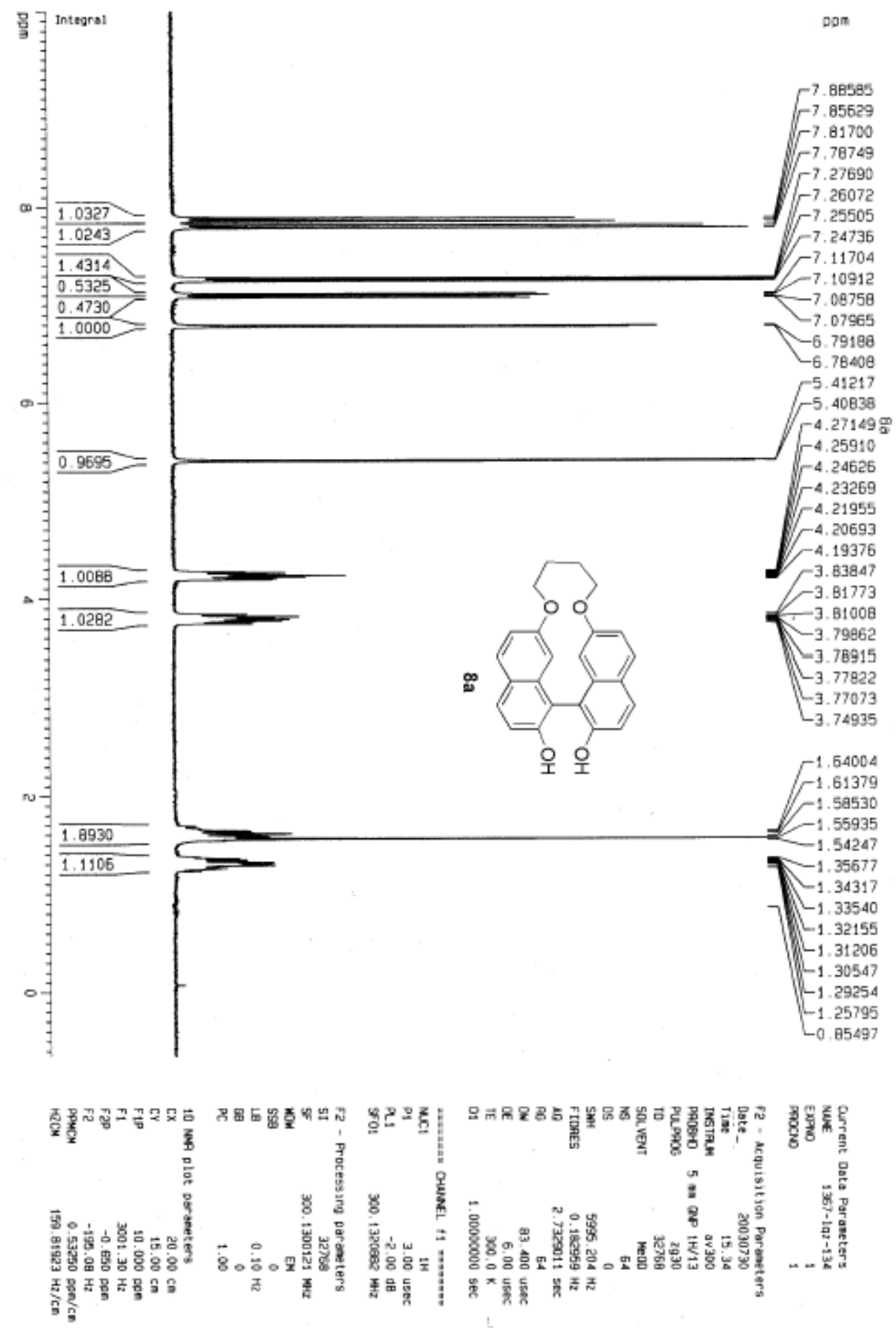
${ }^{13}$ CNMR for compound 8a
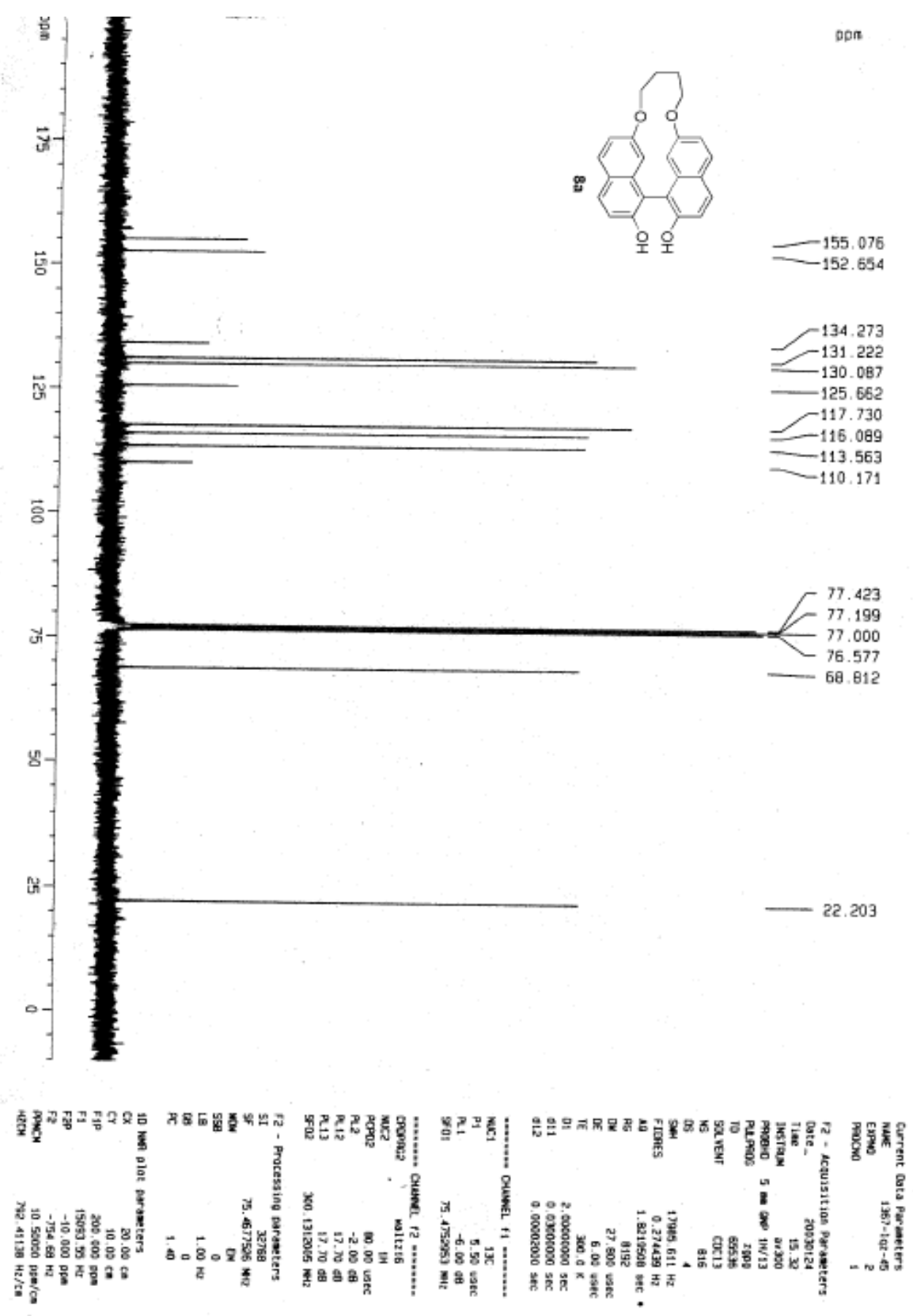
${ }^{1}$ HNMR for compound $\mathbf{8 b}$

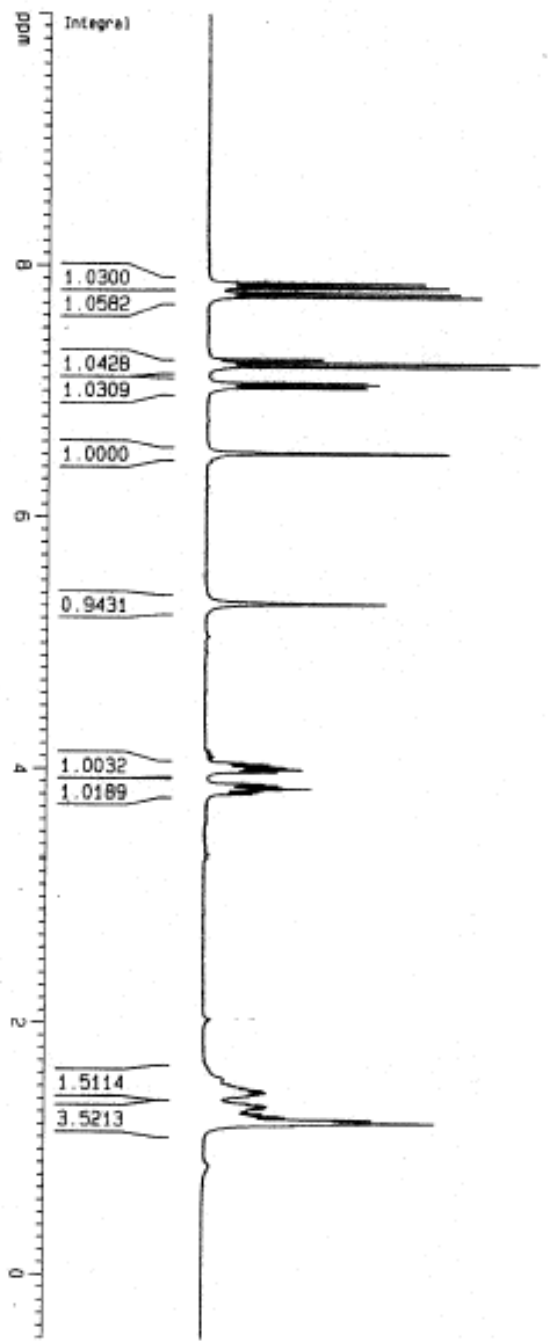

Dpn
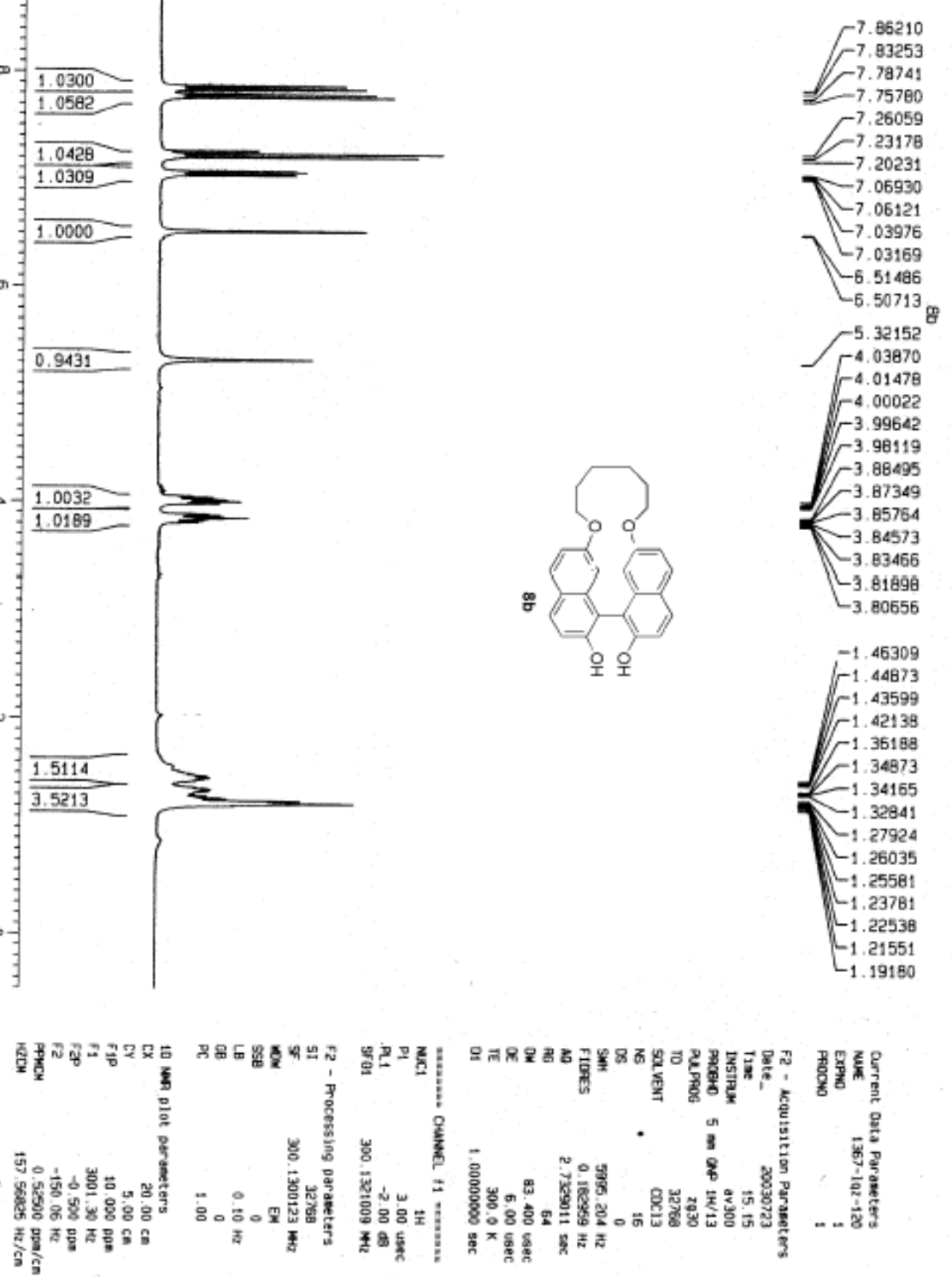
${ }^{13}$ CNMR for compound $\mathbf{8 b}$

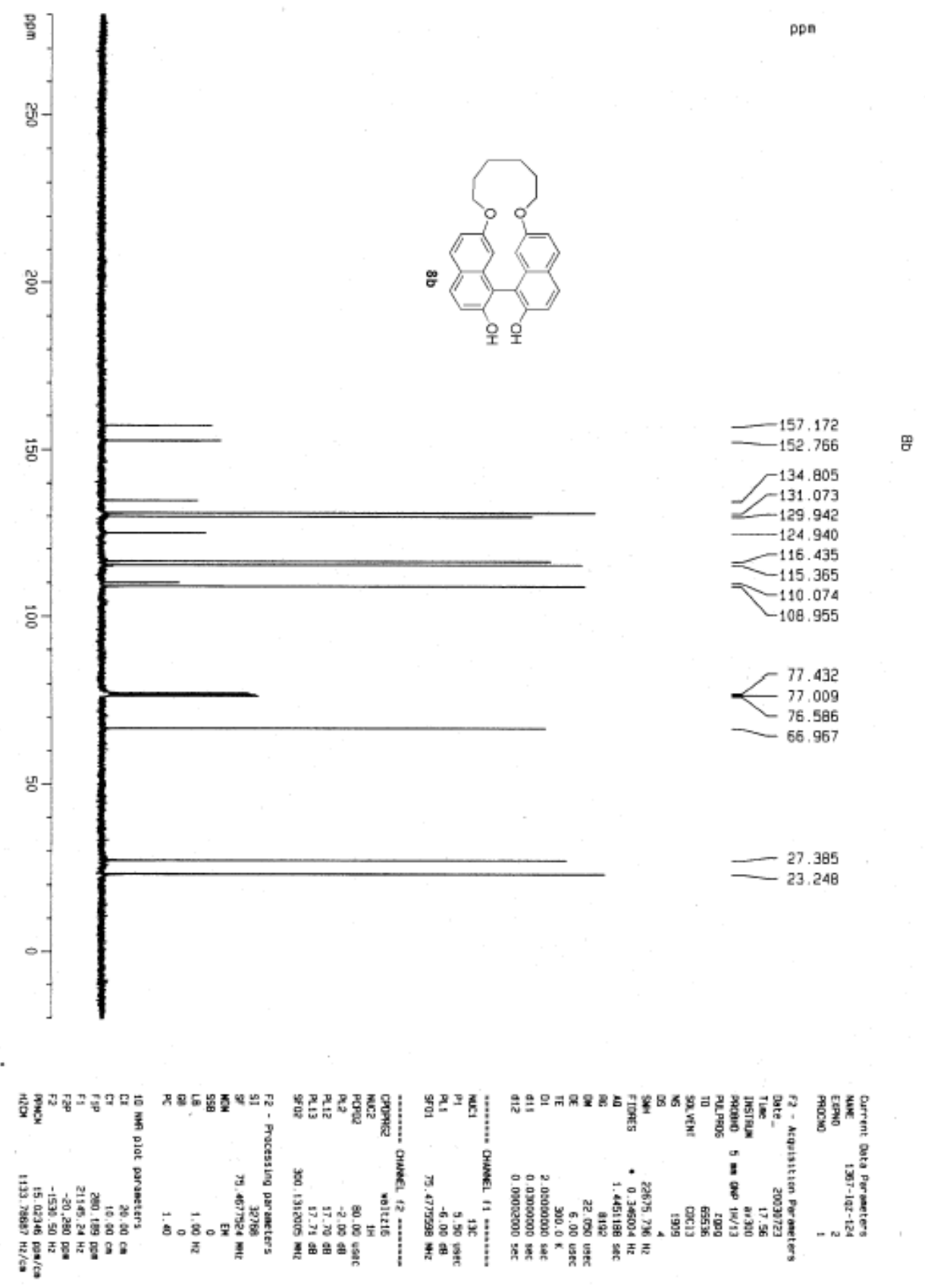


${ }^{1}$ HNMR for compound 8c
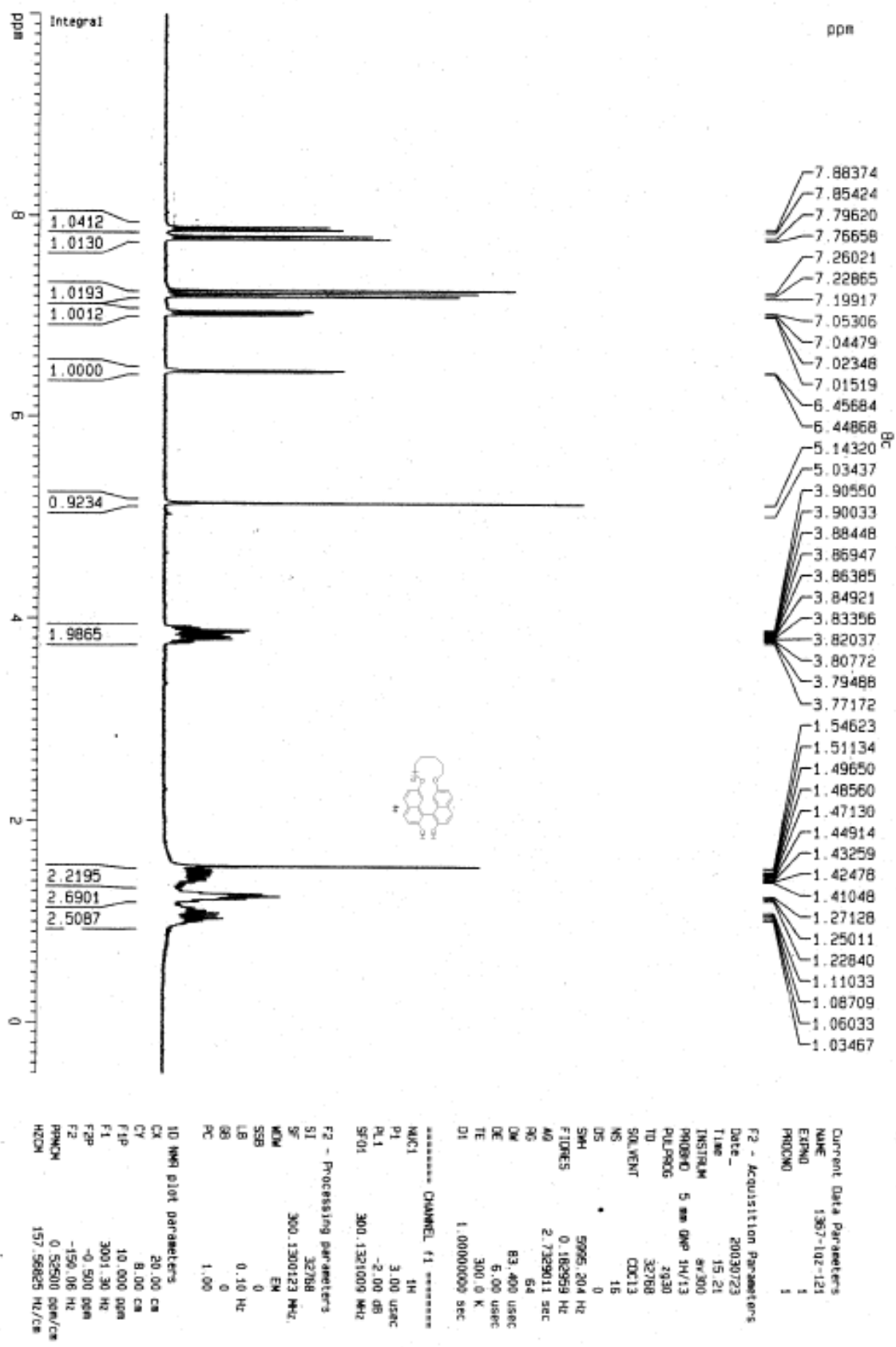
${ }^{13}$ CNMR for compound $8 c$
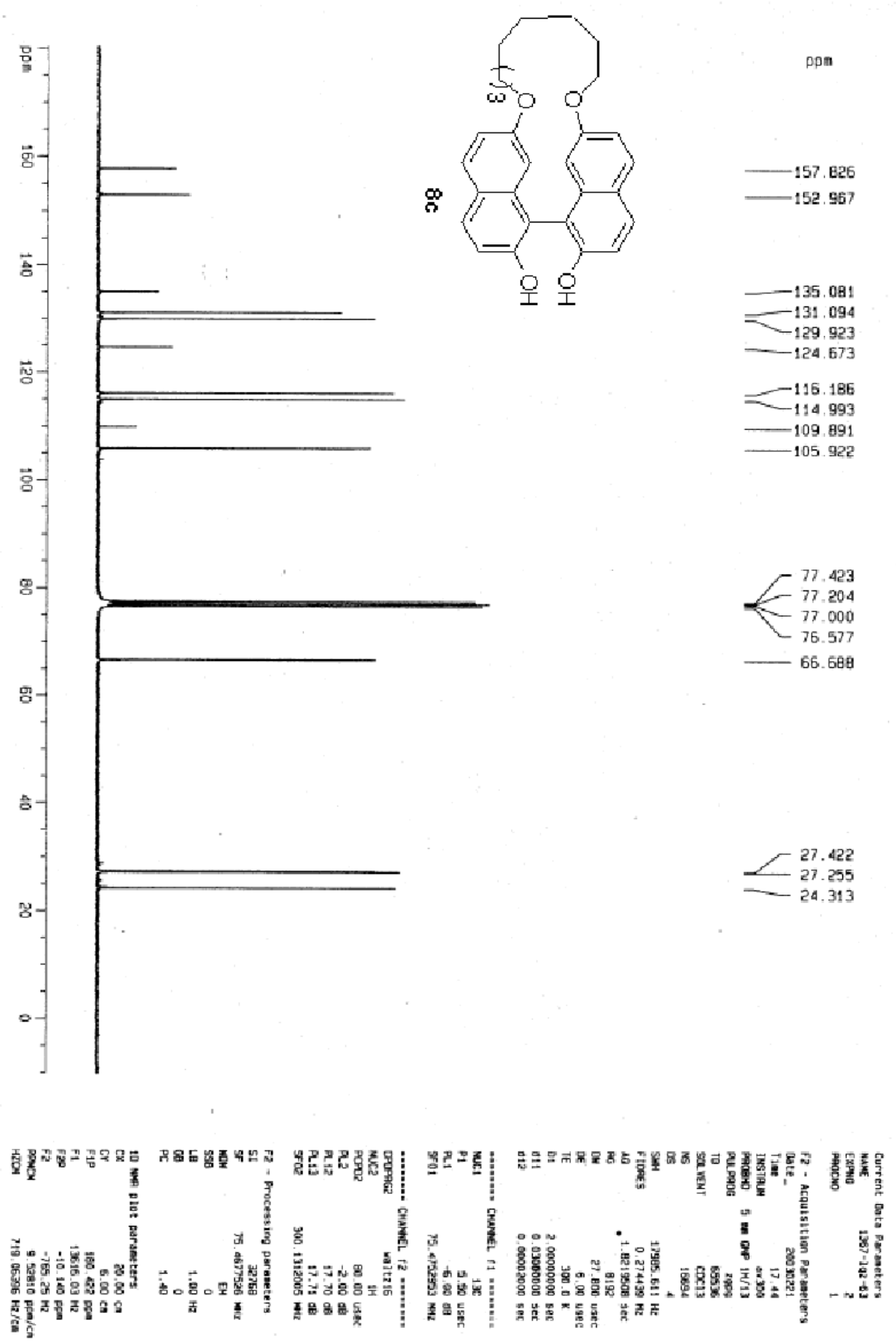
${ }^{1}$ HNMR for compound 9a
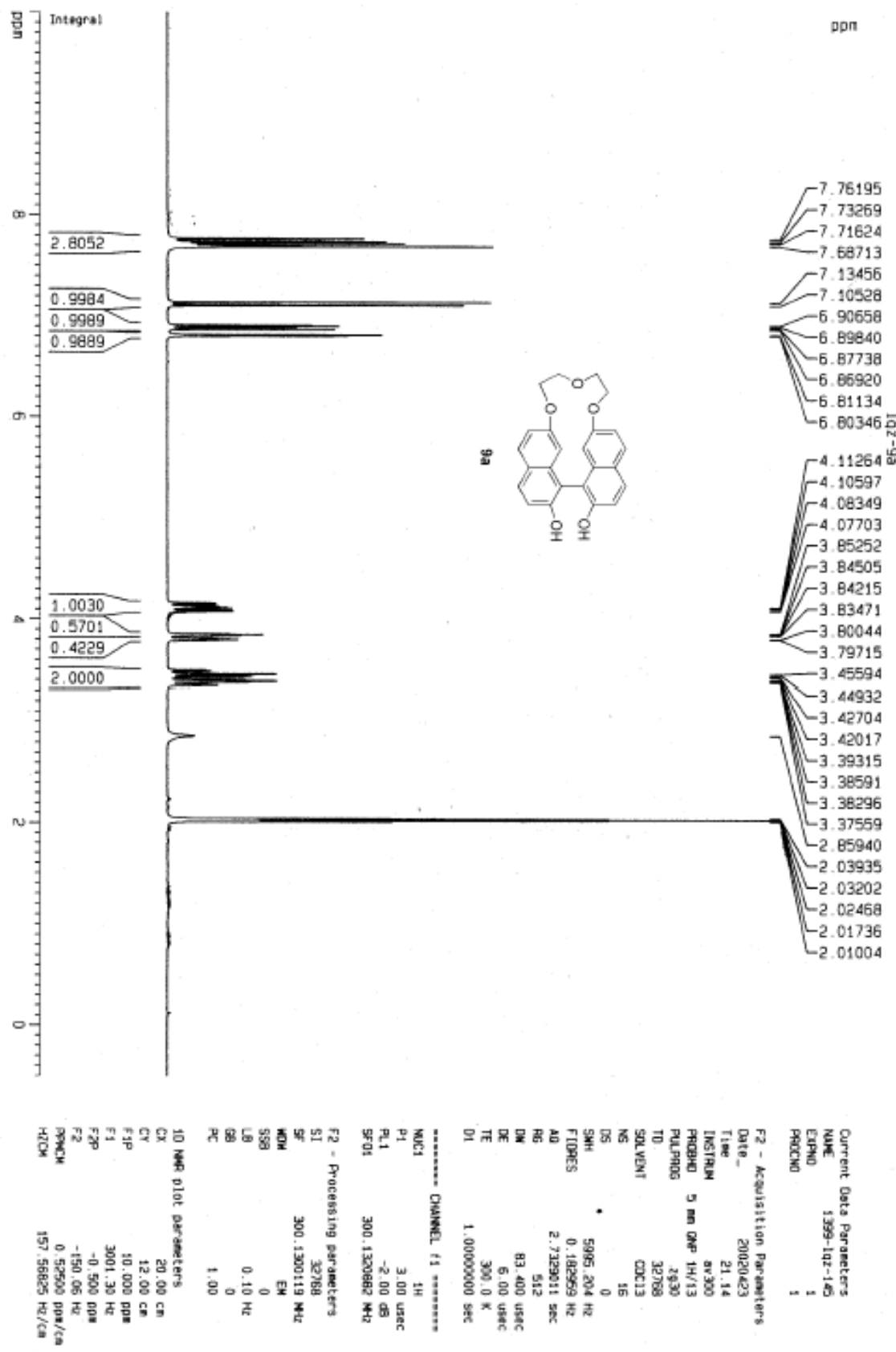
${ }^{13}$ CNMR for compound 9a

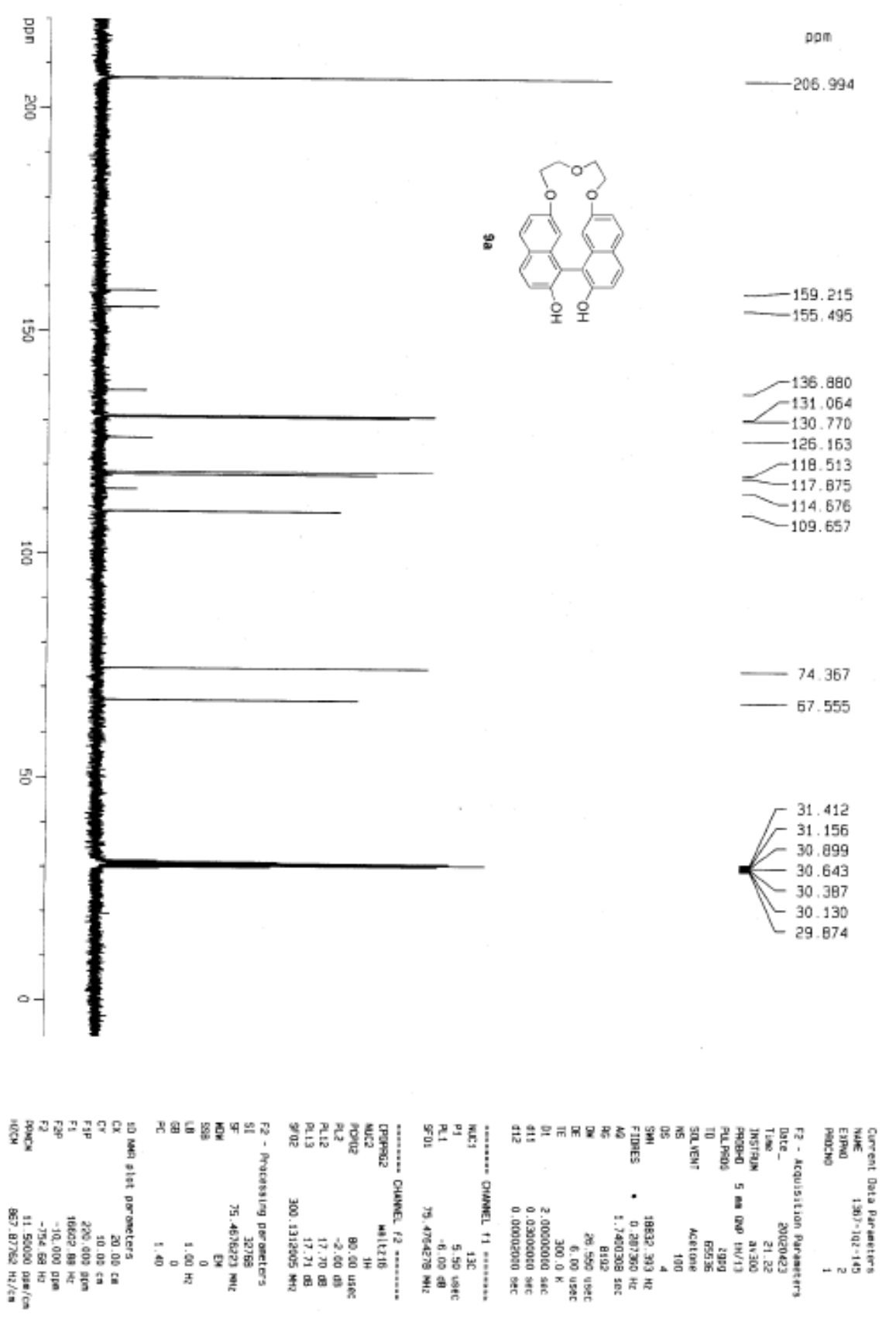


${ }^{1}$ HNMR for compound $\mathbf{9 b}$

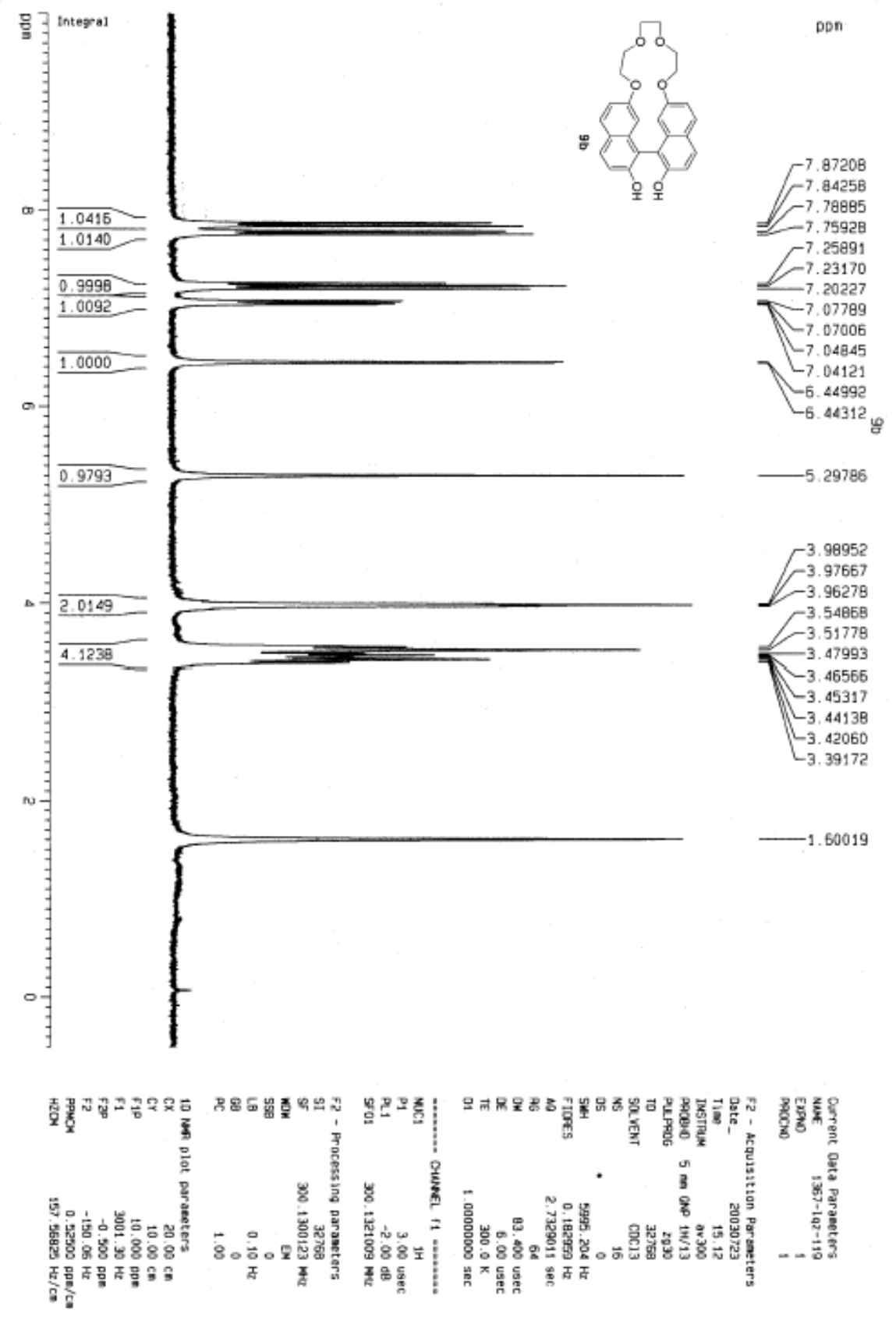


${ }^{13}$ CNMR for compound $9 b$
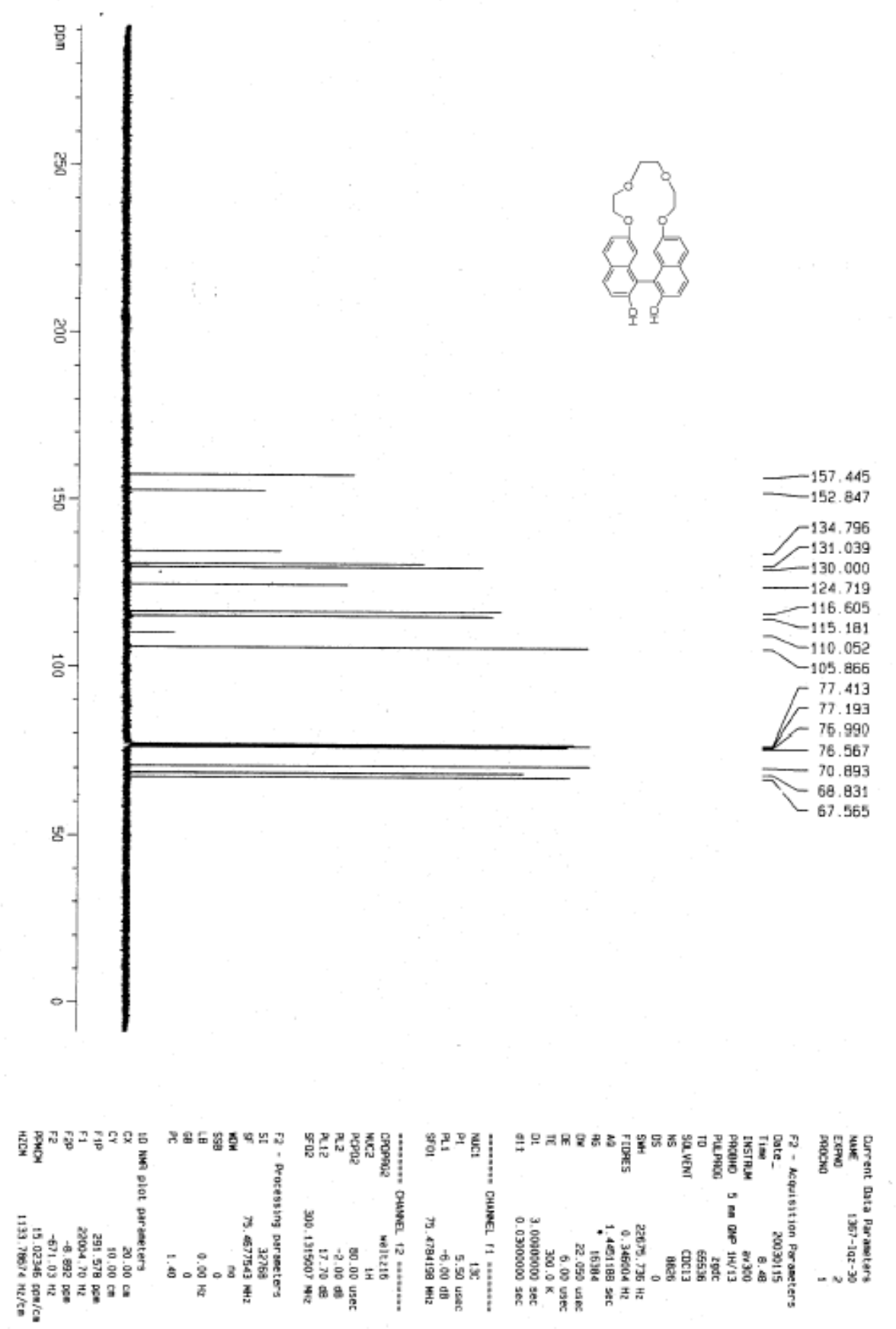
${ }^{1}$ HNMR for compound $\mathbf{1 1}$

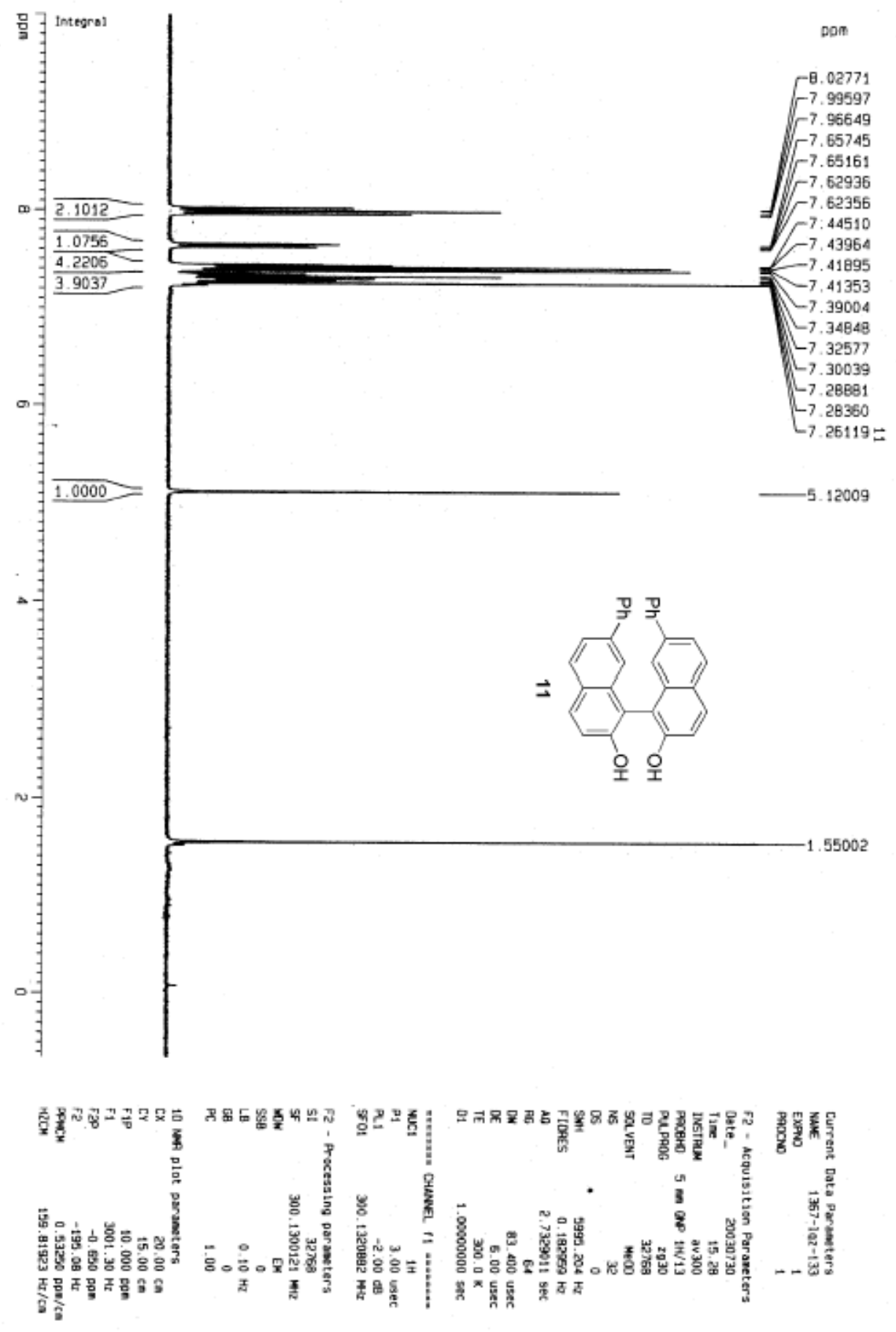


${ }^{13}$ CNMR for compound 11
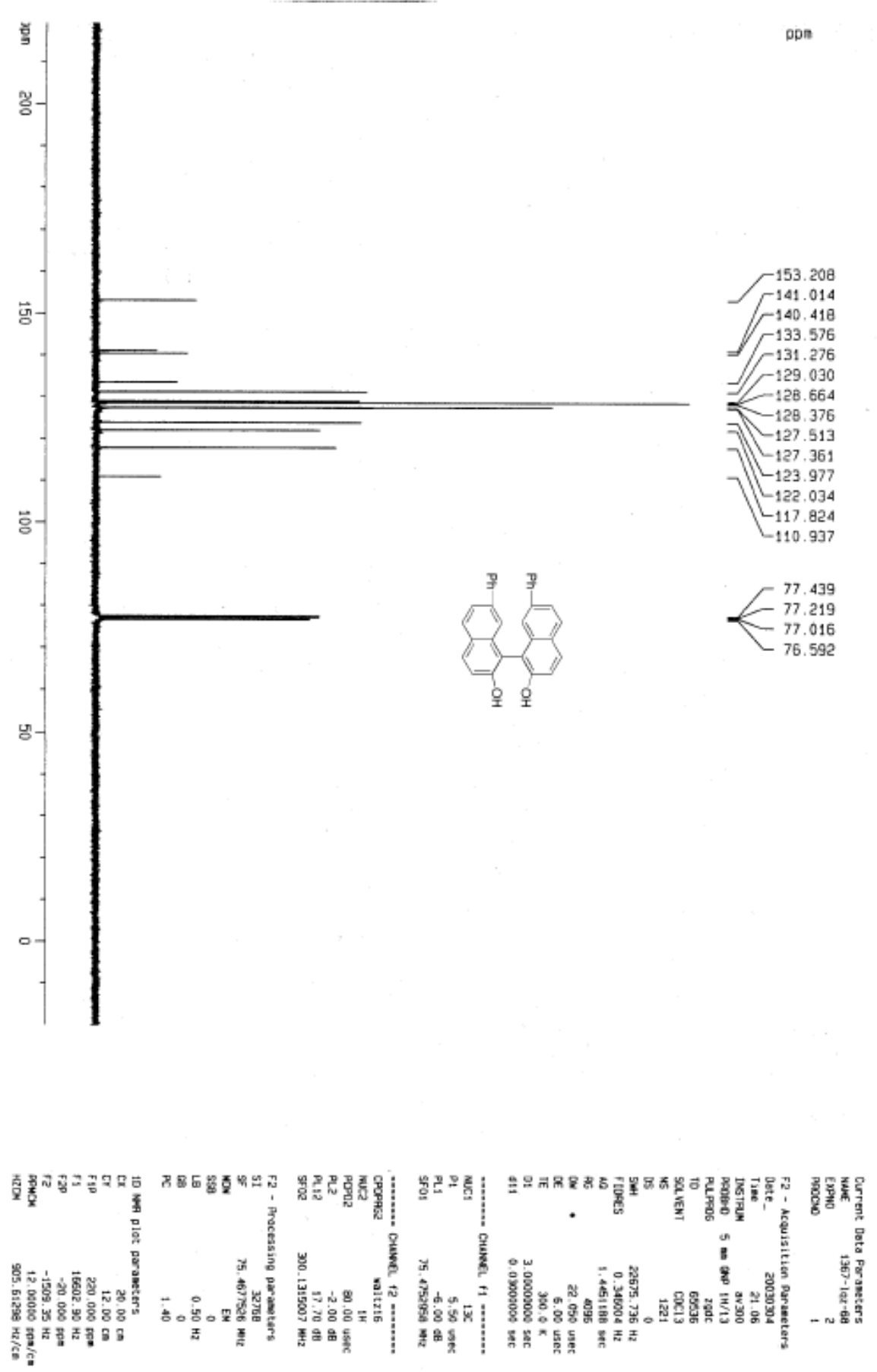